Running title: METASCIENCE MEETS PARAPSYCHOLOGY

\title{
False-Positive Effect in the Radin Double-slit Experiment: HARKing is used by Radin et al. to Misrepresent the Advanced Meta-experimental Protocol used in Walleczek and von Stillfried (2019)
}

\author{
Jan Walleczek and Nikolaus von Stillfried \\ Phenoscience Laboratories, Novalisstrasse 11, Aufgang F \\ Berlin, Germany \\ www.phenoscience.com
}

Keywords: Metascience Reform Movement, Advanced Meta-experimental Protocol, Counterfactual Meta-experimentation, Confirmatory Analysis, HARKing, Radin Double-slit Experiment

Electronic Mail for Corresponding Authors:

walleczek@phenoscience.com

vonstillfried@phenoscience.com

This work was sponsored by the Fetzer Franklin Fund of the John E. Fetzer Memorial Trust 


\begin{abstract}
A general commentary by Walleczek and von Stillfried (2020) was recently published in Frontiers in Psychology. The present work provides an account of (i) the detailed research record and (ii) the main arguments behind the commentary for the purpose of full transparency and disclosure. For historical overview, Walleczek and von Stillfried (2019) had previously reported (i) the absence of any truepositive effects and (ii) the presence of one false-positive effect in a commissioned replication study of the Radin double-slit (DS) experiment on observer consciousness. In their subsequent misrepresentations, Radin et al. $(2019,2020)$ regrettably used the malpractice of undisclosed HARKing, i.e., undisclosed hypothesizing after the results are known. HARKing can increase greatly the risk of false-negative or false-positive conclusions. Specifically, Radin et al. $(2019,2020)$ deviated in two major ways from the pre-specified protocol for this commissioned study, which (i) was agreed to by Radin before data collection was started (Radin, 2011) and (ii) included data encryption to prevent the use of p-hacking and HARKing. First, Radin et al. (2019) violate the original research design by reporting a socalled "true-positive outcome of a secondary planned hypothesis". Contrary to the claim by Radin et al. $(2019,2020)$, that hypothesis was not, however, part of the planned test strategy, but, instead, the associated statistical analysis - a chi-square test - was chosen by Radin sometime after the planned statistical analysis had been completed and the data unblinded. Second, Radin et al. $(2019,2020)$ violate the funder-approved research design in an additional way by falsely claiming that the newly developed protocol, i.e., the advanced meta-experimental protocol (AMP), implements a non-predictive test strategy when - in fact - the AMP-based test strategy is strictly predictive. Put simply, Radin et al. (2019, 2020) are mistaken that the funder-approved hypotheses posited the random occurrence of effects for the test categories in this replication experiment; instead, a different specific prediction was tested in each of the eight planned test categories, and true-positive effects were predicted to occur for only two $(12.5 \%)$ of the 16 possible measurement outcomes of the eight planned single-test categories.

Therefore, in the predictive single-testing regime, a statistical correction for non-predictive, i.e., random, multiple testing would not be appropriate and would thus violate the AMP-based strategy, which was implemented in the commissioned study based upon the planned outcome predictions as pre-specified in Radin (2011). Neither of these post-hoc changes by Radin et al. (on the basis of HARKing) were disclosed in Radin et al. $(2019,2020)$ and both these changes violate the funder-approved, original methodology agreed upon in Radin (2011) and pre-specified in the research contract. In summary, the present work reconfirms that - exactly as reported in Walleczek and von Stillfried (2019) - "the falsepositive effect, which would be indistinguishable from the predicted true-positive effect, was significant at $p=0.021$ ( $\sigma=-2.02 ; N=1,250$ test trials)" and "no statistically significant effects could be identified" in those two groups for which true-positives were predicted to occur. These observations are consistent also with an independent statistical reanalysis of the Radin DS-experiment by Tremblay (2019) and a replication attempt by Guerrer (2019). Tremblay reported significant false-positives in control groups and Guerrer found significant effects with post-hoc analyses only, but null results only when using the planned confirmatory analysis. As a general recommendation, the authors call for the implementation of advanced control-test strategies, including novel approaches from the metascience reform movement, for empirically detecting and preventing uncontrolled false-positive effects in parapsychological research.
\end{abstract}




\section{Introduction}

Walleczek and von Stillfried (2019) previously reported the discovery of a false-positive effect in the Radin double-slit (DS) experiment on observer consciousness with a commissioned replication study. Importantly, the replication study was commissioned to be performed blindly by the same investigator who had reported the original results (Radin et al., 2012). The present work addresses recent criticisms by Radin et al. $(2019,2020)$ claiming that Walleczek and von Stillfried (2019) had failed (i) "to mention that the pre-planned analysis also identified a genuine true-positive result" (Radin et al., 2019) and (ii) to present a proper statistical interpretation. Specifically, it will be clarified that - for both their criticisms - Radin et al. $(2019,2020)$ relied upon unapproved post-hoc changes in the analysis methods, which directly violates the original and funder-approved research agreement (Radin, 2011). That is, Radin et al. $(2019,2020)$ departed from the planned predictive research strategy and - without disclosing this fact - pursued unplanned non-predictive types of analysis - subsequent (i) to having analyzed the original data with the pre-specified protocol, (ii) to breaking the blinding code, and (iii) to examining the decrypted data. This is also known as the malpractice of undisclosed HARKing, i.e., hypothesizing after the results are known (Kerr, 1998). For more explanation, HARKing occurs when a researcher gives the (false) impression that the used form of statistical analysis had been pre-registered, or planned, before unblinding and examining the data, when - in truth - the analysis was developed post-hoc. HARKing increases greatly the risk of mistaking a false discovery for a true discovery or vice versa. Please note that a general commentary article by Walleczek and von Stillfried (2020) was recently published in Frontiers in $\underline{\text { Psychology based }}$ upon the main arguments and research record as will be described in the present work.

To better prevent false discoveries in science in general, the current metascience reform movement seeks to improve the reliability of the scientific process at many different levels (e.g., see www.metascience.com and www.metascience2019.org). For example, the introduction of advanced methodologies which are capable of monitoring, and controlling for, possible violations of best research practices may help improve the reliability of research results. In this context, Walleczek and von Stillfried (2019) have described the use of an advanced methodology known as the advanced meta-experimental protocol (AMP). Specifically, this novel research protocol was employed in the commissioned replication study whose findings are now criticized by Radin et al. $(2019,2020)$. The present work will show that the criticisms by Radin et al. $(2019,2020)$ are unjustified and they violate the confirmatory and strictly predictive test strategy of the AMP-based research design.

In Sect. 2, for the purpose of transparency, the original agreement for implementing this confirmatory and predictive test strategy will be verified by examining the original research proposal by Radin (2011) which was approved by the funder and forms part of the research contract for the commissioned study as reported in Walleczek and von Stillfried (2019). Sects. 3 to 7 will describe in great detail the two major ways in which Radin et al. $(2019,2020)$ - based on the systematic use of HARKing - have deviated from this pre-specified test strategy with the AMP. Sect. 8 will present, for comparison, the results of independent work by other authors relating to the Radin DS-experiment. Finally, Sect. 9 will respond to personal criticisms by Radin et al. $(2019,2020)$ of the funder, including recent false claims regarding post-hoc studies, and Sect. 10 will offer the Conclusions and Outlook.

\section{What was the Confirmatory Predictive Design in the Original Proposal by Radin (2011)?}

A research contract between the Institute of Noetic Sciences (IONS), the host institution of Dean Radin, and the Fetzer Franklin Fund (FFF), the funder who had commissioned the replication study at the IONS, was signed by Dean Radin on October 27, 2011. The research contract refers to a research proposal which - in the signed contract - was titled "Replication of Double-slit Experiment Testing the Effect of Human Intentionality: Adopting a Phase-Four Protocol". The 6-page research proposal (excluding the budget) was written by Dean Radin and was based upon the advanced methodology (AMP) developed by Jan Walleczek. Furthermore, Walleczek is also director of the FFF, and he had both commissioned, and recommended the funding of, this proposal. 
In the following, the original research proposal, which was the basis of the agreement between the IONS and the FFF, will be referred to simply as Radin (2011). In summary, Radin (2011) presented a fully confirmatory replication design which was to be implemented for a replication attempt of prior positive results of a FFF-(co)-funded study (and later published in Radin et al., 2012). Specifically, Radin (2011) credibly presented (1) the goals of the planned task for the confirmatory replication experiment, and (2) the statement of prediction regarding the confirmatory research hypotheses. Next, key passages from Radin (2011) are reproduced, and commented on, in order to clarify the approved parameters of the IONS-FFF research agreement (see Sects. 2.1. to 2.5).

\subsection{Planned Task for the Commissioned Replication Study: Testing for Systematic Errors}

Radin (2011) described the planned task for the commissioned replication study as follows:

"The new task will include an advanced methodology developed by Jan Walleczek to help discern whether the positive results observed in earlier experiments are actually related to aspects of human consciousness (mainly attention and intention), or whether they may have been due to systematic but previously undetected measurement errors." (page 2)

\subsection{True-experimental Protocol: Randomized Experiments With Test Subjects}

The "advanced methodology" (the AMP) reported in Walleczek and von Stillfried (2019) includes an expanded set of experimental and control test categories. In addition to the usually employed experimental test $(X / O)$ where an intervention epoch $(X)$ is compared with a control epoch (O), the AMP employs a number of advanced test categories in the true-experimental protocol, namely the epoch pairs $\mathrm{O} / \mathrm{X}, \mathrm{X} / \mathrm{X}$, and $\mathrm{O} / \mathrm{O}$ (e.g., $\mathrm{X}=$ attention; $\mathrm{O}=$ attention withdrawn), whereby $\mathrm{O} / \mathrm{X}$ is the systematic time-reversed control (STC), $\mathrm{X} / \mathrm{X}$ is the systematic positive control (SPC), and O/O is the systematic negative control (SNC). Critically, for the FFF-approved research proposal, Radin (2011) had defined (i) the statement of prediction, and (ii) the confirmatory and blinded (encrypted) design, as follows:

"The hypothesis is that comparisons between epochs with $\mathrm{X}-\mathrm{O}$ or $\mathrm{O}-\mathrm{X}$ comparisons should show a genuine difference, but $X-X$ and $0-O$ comparisons should show no difference." (page 4)

"Prior to the experiment a computer will randomly assign the four epochpair conditions (XX, OO, XO, and OX) to the letters A, B, C, or D. A third-party will hold these letter-condition codes to keep the experimenters blinded to the experimental conditions during the data collection process. The code will be broken only after all data from all 25 subjects (a total of 250 experimental and 250 sham sessions) has been collected and analyzed." (page 5)

Note that in the above statement of prediction by Radin (2011), predictive hypothesis testing is based upon the statistical comparison of collected population means $X$ and $O$ between epochs (e.g., "between epochs with X-O") but not for any X- or O-means between different epoch pair conditions. For a tutorial example explaining the statistical interpretation in the predictive single-testing strategy see Sect. 5.1. For agreement between this original statement of prediction by Radin (2011) and the predictions in Walleczek and von Stillfried (2019), see also Fig. 3A in Sect. 7.2.

\subsection{Sham-experimental Protocol: Randomized Experiments Without Test Subjects}

In addition to the above described advanced control test categories in the trueexperimental protocol, the AMP reported in Walleczek and von Stillfried (2019) also implemented a 
sham-experimental protocol. Radin (2011) correctly described its nature and its pre-specified purpose in relation to the true-experimental protocol as follows:

"The companion 'sham' protocol will be a control test consisting of the same protocol, but without subjects present." Specifically, after each (true)experimental session (see Sect. 2.2), “... an identical session will be run as a sham control using the same epoch-pair orders used in the experimental session, except no one will be present." (page 4)

"This method is evaluated exclusively with epoch-pair differences because the aim is to test the effect of systematic fluctuations in the data (e.g., linear drifts or regular oscillations), and to ensure that the experimental intervention (in our case the act of focusing attention towards the doubleslit system) has a real effect." (page 4)

Briefly, regarding the four epoch pairs for the sham-experimental hypotheses $\left(\mathrm{X}_{S} / \mathrm{O}_{s}, \mathrm{O}_{s} / \mathrm{O}_{s}\right.$, $\mathrm{X}_{\mathrm{S}} / \mathrm{X}_{\mathrm{s}}$, and $\mathrm{O}_{\mathrm{s}} / \mathrm{X}_{\mathrm{s}}$; see Sect. 7.3 for Fig. $4 \mathrm{~B}$ ), only two of the eight possible outcomes (including increase and decrease effects) are predicted to constitute a false-positive effect that could be mistaken for the (predicted) "real effect" (Radin, 2011). See Sect. 7.3. for more explanations.

\subsection{The Predicted Outcome Measure is a Decrease in Double-slit Light Interference Intensity}

Radin (2011) wrote that "The variable of interest in this experiment is the peak spectral power associated with the double-slit component of the interference pattern produced by an optical double-slit apparatus." Specifically, the "... 'consciousness collapse' hypothesis predicts... that... during concentrated attention the spectral power associated with a double-slit is expected to decline with respect to the same measurement recorded when attention is withdrawn." (page 3)

\subsection{Summary of the Confirmatory Design in Radin (2011) and the Misrepresentation}

The two main goals of the FFF-commissioned replication study - as originally described by Radin (2011) - can be framed as two distinct research questions in relation to Radin et al. (2012):

(1) Can the light-intensity decrease effect reported in Radin et al. (2012) be replicated at the IONS by Radin using a blinding protocol implementing a confirmatory and predictive design?

(2) Can the (hidden) presence of "systematic but previously undetected errors", e.g., a falsepositive effect, be ruled out as an obvious explanation for the light-intensity decrease effect which was reported in Radin et al. (2012)?

Briefly, after unblinding the "collected and analyzed" data (Sect. 2.2) on March 11, 2013 - in the presence of both Radin and Walleczek at the IONS - the answer to both questions was found to be 'No'. It is these findings that were published in Walleczek and von Stillfried (2019).

Summarizing the time line, (i) the research proposal by Radin (2011), which presented the planned research tasks and predictions (Sects. 2.1. to 2.4), was submitted to, and approved by, Walleczek in October 2011, (ii) data collection with the advanced methodology commenced in 2012, and finally, (iii) the data were statistically analyzed, and then unblinded - in the presence of both Radin and Walleczek - on March 11, 2013 (see also Fig. 1B in Sect. 3.1). After a thorough review and independent analysis of the statistical routines and the used data interpretation in this confirmatory replication study, these results were eventually published by Walleczek and von Stillfried (2019). Importantly, this independent analysis was performed at Phenoscience Laboratories in Berlin (www.phenoscience.com) with the expressed permission, and approval, of the President of the 
IONS, which, again, is the host institution of Radin. The present work will demonstrate that the results published in Walleczek and von Stillfried (2019) are in exact agreement with the confirmatory, predictive methodology as was described in Radin (2011).

Nevertheless, in violation of the funder-approved research and publication process, Radin et al. $(2019,2020)$ chose to ignore the confirmatory and predictive AMP-based research design which had been committed to in the original and FFF-funded proposal by Radin (2011), and which was used by Walleczek and von Stillfried (2019). Instead, Radin et al. $(2019,2020)$ chose to misrepresent the AMP-based results by way of promoting an unapproved post-hoc, i.e., unplanned, statistical interpretation based on the malpractice of HARKing.

The reader who is interested in the details concerning the exact way in which Radin et al. $(2019,2020)$ departed from the agreed upon protocol may consult Sects. 3 to 7 . It is unfortunate that this admittedly extensive amount of background information is necessary; however, given the elaborate construction - based on HARKing - of the misrepresentation by Radin et al. $(2019,2020)$, there is no other good way to clarify these important issues. The reader who is not interested in these details might advance directly to Sects. 8 to 10.

Sect. 3 will show that the claim by Radin et al. (2019) of a "true-positive outcome of a secondary planned analysis" is false because that outcome - in truth - resulted from an unplanned statistical analysis - a chi-square test - based on the harmful use of HARKing (Kerr, 1998).

\section{On the False Claim by Radin et al. (2019) of a "True-positive Outcome of a Secondary Planned Analysis" in the Commissioned Replication Study}

Radin et al. (2019) claimed the identification of a true-positive outcome with a secondary planned analysis, and they wrote in the abstract: "Walleczek \& von Stillfried did not report a truepositive outcome of a secondary planned hypothesis that remained significant after adjustment for multiple testing." However, the claim by Radin et al. $(2019,2020)$ of the so-called "true-positive outcome" is simply false. After disclosing the correct research record and time line further below, it will become evident that the so-called "true-positive outcome" (Radin et al., 2019, 2020) was not the result of any "secondary planned hypothesis" or "pre-planned analysis". To the contrary, the socalled "true-positive outcome" was derived from a form of statistical hypothesis testing that was developed post-hoc, i.e., after Radin had first viewed the results on March 11, 2013. Therefore, when Radin et al. (2019) accuse Walleczek and von Stillfried (2019) of failing "to mention that the pre-planned analysis also identified a genuine true-positive result", their false assertion is not based on the known facts of this research project and directly contradicts the well-documented research record for the commissioned replication study.

The subsequent Sects. 3.1 to 3.6 will document that Radin has performed - at least - three different forms of statistical analysis - two of which were based on the malpractice of HARKing. These three different statistical analyses yielded mutually incompatible experimental conclusions for the observer-consciousness effect in the true-experimental test categories $\mathrm{X} / \mathrm{O}$ and $\mathrm{O} / \mathrm{X}$ (Sect. 2.2): (1) a complete null result for both directional and non-directional analyses (Radin, 2013; see Fig. 1B in Sect. 3.1, and Sect. 3.3 regarding the correction for multiple testing), (2) a directional decrease effect (Radin et al., 2013; see Fig. 2 in Sect. 3.2) and (3) a non-directional effect with a chisquare test analysis (Radin et al., 2019; see Fig. 1A in Sect. 3.1).

Regrettably, the history and the precise nature of these contradictory outcomes ( 1 to 3 ) has neither been disclosed in Radin et al. (2019) nor in Radin et al. (2020). Instead, Radin et al. (2020) chose to accuse one of the co-authors of Walleczek and von Stillfried (2019) of suppressing scientific information when Radin et al. (2020) wrote that "... we did not publish the results of that experiment at Walleczek's request". In the following, the actual research history will be documented in detail in 
Sects. 3.1 to 3.6 in order to clarify for the reader how Radin et al. $(2019,2020)$ have constructed their false narrative for misrepresenting the AMP-based commissioned replication study.

\subsection{The So-called Secondary "Pre-planned Analysis" was Developed Post-Hoc (HARKing)}

The results from any "planned hypothesis" could only be those that were revealed on the day of breaking the blinding code (March 11, 2013). This unblinding happened in the physical presence of both Radin and Walleczek at the IONS, whereby the latter held the decryption key for breaking the blinding code (see also Sect. 7).

Importantly, for the purpose of independent documentation of the results as were obtained with the "pre-planned analysis" after unblinding, Radin had forwarded - on March 11, 2013 - to Walleczek a digital copy of these decrypted results in the form of a power-point presentation (Radin, 2013), which listed the complete set of statistical findings for all of the planned tests including planned single tests and planned multiple tests (see also Sect. 3.3) The availability of this digital copy now allows for the objective comparison between the original results of the "preplanned analysis" on March 11, 2013 (see Fig. 1B) and the so-called "true-positive" result now claimed by Radin et al. $(2019,2020)$ for the variance-type analysis in the specific form of the chisquare test in the table (see the value of 309.5, at $p=0.006$; Fig. 1A) from Radin et al. (2019).

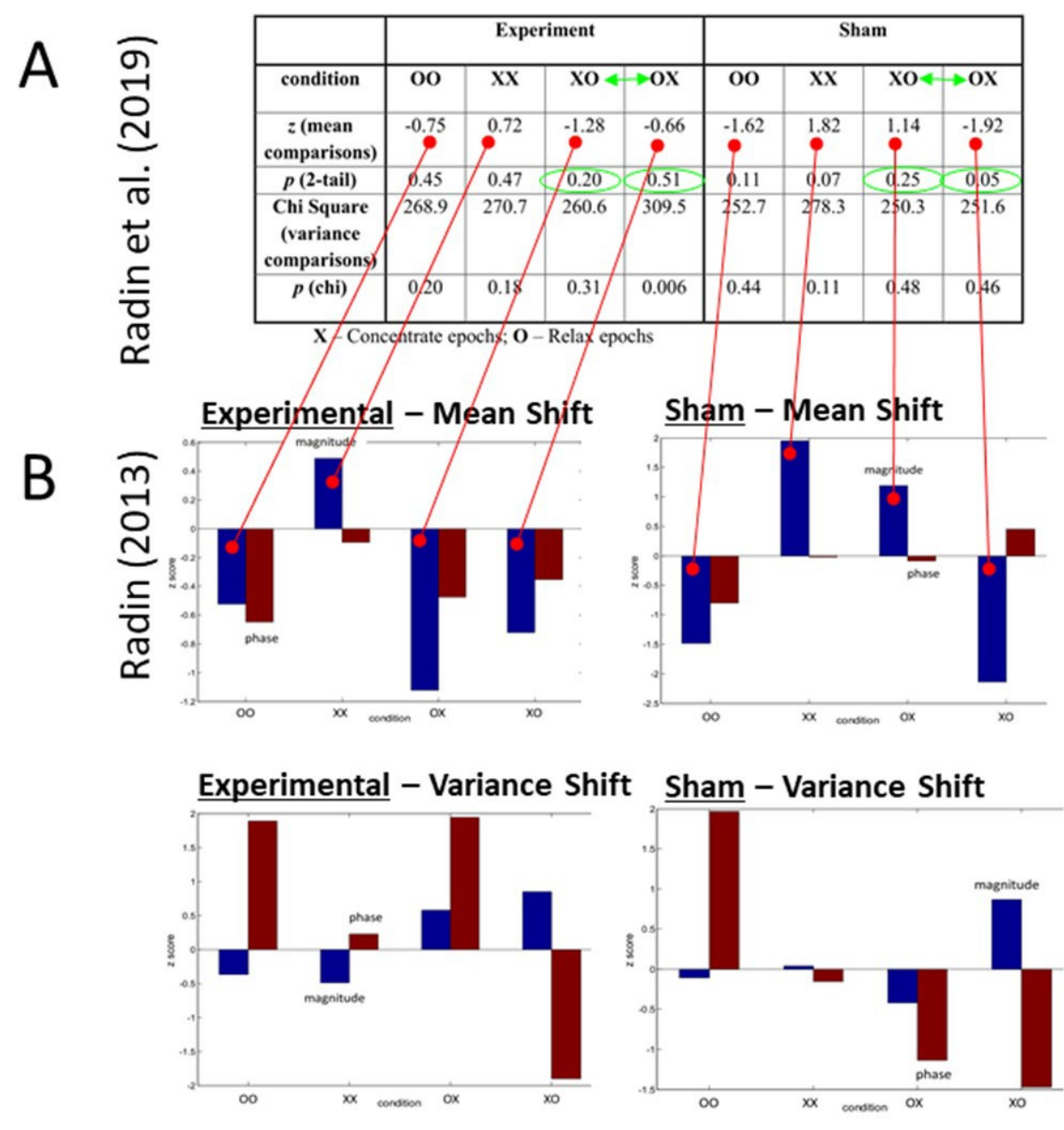

Figure 1: Comparison of the results presented in Radin et al. (2019) in Fig. 1A with the results of the original analysis after breaking the blinding code on March 11, 2013 in Fig. 1B. A comparison (dots + lines) first reveals that Radin et al. (2019) reports only one fourth of the original results, as explained in Sect. 3.3. (Concerning an explanation of the minor differences between the graphically displayed 
data in Radin, 2013, and the numeric values in Radin et al., 2019, consult Sect. 7). Second, it can be seen that the results for the chi-square test (Fig. 1A) reported in Radin et al. (2019) are not present as part of the original results (Fig. 1B) as documented in Radin (2013). Please note also that twotailed tests were (falsely) calculated (see ellipsoids) in Radin et al. (2019) whereas one-tailed tests would have been required due to the directional hypotheses pre-specified in the original research proposal (Radin, 2011) and that certain column labels (see double-arrows) were falsely assigned in Radin et al. (2019). This comparison is for versions 1 to 4 of the pre-print by Radin et al. (2019).

After comparing the results in Figs. $1 \mathrm{~A}$ and $1 \mathrm{~B}$, it is evident that a chi-square test was not programmed in the original Matlab script that was used for the blinded statistical analysis on March 11, 2013. For further explanation, the direct comparison of the results displayed in Fig. 1

demonstrates that none of the statistical findings for the planned variance analysis (Fig. 1B; for more details see also Sect. 3.3) as performed on March 11, 2013 (Radin, 2013) agree with those for the (unplanned) chi-square test analysis as now reported by Radin et al. (2019) in their Table 1 (Fig. 1A). Put simply, the claim by Radin et al. (2019) that "the pre-planned analysis also identified a genuine true-positive result" is demonstrably false, because the so-called "true-positive" result is not a function of the "pre-planned analysis" but - instead - is a function of HARKing, i.e., of undisclosed hypothesizing after the results were known (Kerr, 1998).

The decision by Radin et al. (2019) to abandon the planned variance analysis (see Fig. 1B; variance shift), which found null results only (for details see Sect. 3.3), in favor of another kind of bidirectional analysis based on a chi-square test (see Fig. 1A) conforms to a version of HARKing that was described by Kerr (1998) under the heading "Empirical Inspiration". For one example, Kerr explained that:

“... in an approach we might term re-evaluation of theory, empirical disconfirmation of the original hypothesis might prompt re-examination of your original theoretical logic... with the result that you conclude that the theory really should have led you to a hypothesis rather different from the one originally conceived and more consistent with current data."

(page 198)

It is apparent that Radin et al. $(2019,2020)$ have changed the analytic methodology sometime after having unblinded the results on March 11, 2013. Most importantly, there exists no evidence for a pre-registration of this chi-square test analysis, and the funder had neither been informed nor had ever approved such a chi-square test analysis; in fact, the funder found out about the results with the chi-square test analysis only after Radin had posted a pre-print on psyarxiv.com in October 2019 (Radin et al., 2019) - more than six years after the original unblinding of the results in March 2013, and only after the publication by Walleczek and von Stillfried (2019), which is now criticized.

Crucially, if these results existed - in fact - already on March 11, 2013, then the funder of the commissioned study was kept willfully in the dark about these findings for more than six years, in violation of the research contract between the IONS and the FFF. Even as recently as June 2018, none of the official progress reports, or any of the other written statements received by the funder from Radin, had ever mentioned - not with a single word - the claim of a significant bi-directional effect based on a chi-square test analysis, which is now the basis of the criticism by Radin et al. $(2019,2020)$.

In conclusion, whatever the relevance of the so-called "true-positive result" may be, this claimed ("true-positive") result - contrary to the claim by Radin et al. (2019) - was not due to a "secondary planned analysis" which had supposedly been performed on March 11, 2013. What 
follows next is the disclosure of additional information - concerning the malpractice of HARKing from the detailed research record for this funder-commissioned replication study of the Radin DS- experiment.

Between 2013 and 2018, Radin suggested different types of analysis in relation to the same collected raw data set, which included unapproved post-hoc alterations in the tested hypotheses and forms of statistical analysis, and this violated the funder-approved research agreement (for comparison see Sect. 2.2.). This essential information regarding the post-hoc changes to the original, fully blinded form of analysis has not been disclosed in Radin et al. $(2019,2020)$. Again, the present manuscript seeks to remedy this situation by providing background information regarding the violation by Radin et al. of the funder-approved scientific process for the commissioned study.

The information in subsequent Sect. 3.2. will demonstrate that - already in September 2013 - Radin had used yet another post-hoc adapted form of analysis, in violation of the planned form of analysis, in an apparent attempt to turn the original null effect for the directional mean-difference measure (see Fig. 1B) into a seemingly positive directional effect (see Fig. 2 below).

\subsection{A Directional Observer Effect is Reported by Radin et al. (2013): Progress Report to theFFF}

In September 2013, Radin et al. (2013) submitted to the funder (FFF), a progress report in the form of a draft manuscript for publication, which stated the following entirely directional hypothesis, namely the prediction of a decline effect due to observer consciousness:

"The hypothesis was the same as in previous experiments - that the doubleslit spectral component of the interference pattern would decline during observation periods as compared to a no-observation control periods." (page 8)

That progress report - in the form of a proposed manuscript for publication (Radin et al., 2013) - was received about six months after the original data analysis and unblinding of the results at the IONS had found null results only for the predicted test categories $X / O$ and $O / X$ in the presence of observer consciousness (for these original null results see Fig. 1B above). However, contrary to the null results of the original, blinded analysis submitted to the funder on March 11, 2013 (see Fig. $1 B)$, this draft manuscript - to the great surprise of the funder of this commissioned study reported now a statistically-significant directional observer consciousness effect. See Fig. 2 for the two true-experimental test categories $\mathrm{O} / \mathrm{X}$ and X/O, for which Radin et al. (2013) claimed a significant difference at $p=0.03$ by means of a t-test.

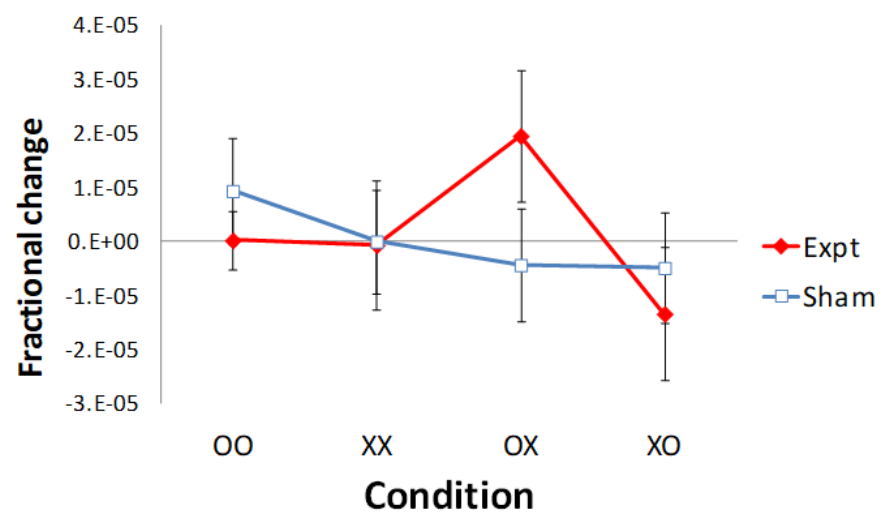

Figure 2: This figure is reproduced from the draft manuscript submitted to the funder by Radin et al. in September 2013 as a progress report (Radin et al., 2013). See the main text for more details regarding this claimed directional effect by Radin et al. (2013). It is astonishing that the same 
investigators now report that a directional effect had not been claimed for this experiment (Radin et al., 2019, 2020).

It was this new claim of a directional effect as shown in Fig. 2, which finally caused the funder to request of the leadership at the IONS to review and verify - prior to submission of this result for publication - whether the funder-approved AMP-based test strategy had possibly been compromised by either p-hacking or HARKing, given the contradictory nature of the results; by contrast, incomprehensively, Radin et al. $(2019,2020)$ recently promoted the false narrative that it was the non-directional effect based on the chi-square analysis which had been requested by the funder to not be published. However, how could that request have been made by the funder, when the funder - as had already been mentioned - had only found out about the novel claim regarding a non-directional effect in October 2019, when a pre-print was published on psyarxiv.com (Radin et al., 2019)? The accusation by Radin does not make any sense, and is not supported by the record.

Crucially, the claim by Radin et al. (2019) that this result had already been known as part of the original analysis in 2013 (in March 2013 or even a post-hoc analysis in September 2013) is false: there is no agreement between the claimed results in the form of the proposed manuscript that was submitted to the funder in September 2013 (Radin et al., 2013), or also the original analysis in March 2013 (Radin, 2013), and the so-called "true-positive" effect now suggested in Radin et al. (2019), which claimed a bi-directional effect based on a chi-square test analysis (see Figs. 1 and2).

Concluding, there is neither an agreement between the results from the chi-square test analysis (Radin et al, 2019) and the original results as obtained in March 2013 (Radin, 2013), nor is there an agreement between the results from the chi-square test (Radin et al., 2019) and the results reported to the funder in September 2013 (Radin et al., 2013). Again, remarkably, none of this has been disclosed in Radin et al. $(2019,2020)$, and this lack of transparency is raising more questions.

\subsection{The Original Matlab-based Statistical Analysis Involved Four Planned Outcome Measures Whereby Three of Those Measures were not Pre-specified}

For the purpose of transparency, the following essential information should also be fully disclosed because Radin et al. $(2019,2020)$ have failed to do so in their recent critique of Walleczek and von Stillfried (2019): Besides the analysis of the pre-specified outcome measure (see Radin, 2011), which was the predicted decrease in DS-light interference intensity (see Sect. 2.4), Radin had also programmed in the original Matlab-based script - on his own initiative - three additional types of analyses, concerning three additional outcome measures, which had not been pre-specified in the original research proposal in Radin (2011). It cannot - obviously - be under the control of the funder what additional types of outcome measures, which were entirely unrelated to the FFF-commissioned replication attempt, Radin and co-workers may, or may not, choose to program in the Matlab script.

Specifically, the additional analyses as documented in Radin (2013) were: (i) a phase measure in relation to the mean-shift measure, (ii) a magnitude measure in relation to a varianceshift measure, and (iii) a phase measure in relation to a variance-shift measure. For an overview of all these original results see Fig. 1B. Please note that - as will be explained further in Sect. 3.5 none of the variance-shift measures in the original Matlab script (Radin, 2013) equal the measure based on the (post-hoc) chi-square test analysis as reported in Radin et al. (2019). Compare also Figs. $1 \mathrm{~A}$ and $1 \mathrm{~B}$.

In summary, the three additional outcome measures did not seek to confirm the "positive results observed in earlier experiments" (Radin, 2011). In any case, in contrast to the pre-specified outcome measure, which was analyzed using the planned single-testing strategy (see also Sect. 4), the three additional outcome measures were part of a planned multiple-testing strategy (for an overview see the table in Sect. 3.5). 
Finally, any possible significant outcome for any of the three additional measures must be judged against the calculated threshold for significance testing after a statistical correction for (planned) multiple testing: None of the results with these three new measures crosses the threshold for statistical significance. This negative result for the non-directional variance analysis with the planned, multiple-testing strategy (Radin, 2013) was not even mentioned in Radin et al. (2019); instead, Radin et al. (2019) constructed a false narrative for a so-called "true-positive result".

\subsection{Constructing the False Narrative for a "Genuine True-positive Result" (Radin et al.,2019)}

Radin et al. (2019) chose to construct the false narrative that a "pre-planned analysis also identified a genuine true-positive result" (see also Sect. 3.1). Furthermore, based on that false narrative, Radin et al. (2019) accused Walleczek and von Stillfried (2019) of suppressing the report of a "genuine true-positive result", when Radin et al. (2019) wrote:

"The terms "pre-specified" and "pre-planned" appeared 32 times in WS's article, emphasizing their contention that the methods of analysis were strictly established prior to decryption of the attention conditions. This design feature was included to prevent post-hoc $p$-hacking or data snooping. Given that emphasis, it is surprising that these analyses were not described in WS's paper. Instead, they wrote, "For viewing the technical details of the employed signal processing routines, this original Matlab script ... can be made available upon request" (WS, p. 4). When the script they provide is examined, it is found to include both the mean and the variance analyses shown above in Table 1. This indicates that WS emphasized an unwarranted false-positive outcome but failed to mention that the pre-planned analysis also identified a genuine true-positive result." (page 19)

As was already explained in Sect. 3.1., contrary to false narrative by Radin et al. (2019), the so-called "pre-planned analysis" was not at all "pre-planned" but was developed post-hoc by Radin. Therefore, it should not be "surprising that these analyses were not described in WS's paper"; only the results of the confirmatory replication study, i.e., of the study that was pre-specified in the original, funder-approved research proposal (Radin, 2011), were reported in Walleczek and von Stillfried (2019). This was done in accord with strictly confirmatory principles - in order "to prevent post-hoc p-hacking or data snooping" (and HARKing; see Sect. 3). It should also be clarified that $\underline{\text { Table } 1} \underline{1}$ in Radin et al. (2019) has mislabeled the relevant conditions for the mean difference analysis in four separate positions: For the correct representation, the labels for conditions " $\mathrm{X} / \mathrm{O}$ " and "O/X" must be switched for both "Experiment" and "Sham" (see also the legend to Fig. 1).

The last remaining question is the following then: Why did the Matlab script that was received by Radin from Walleczek and von Stillfried contain an unplanned statistical analysis, even though only planned analyses were performed according to Walleczek and von Stillfried (2019)? As will be shown in Sect. 3.5, the simple answer is that this Matlab script was not the one that was originally used on March 11, 2013.

\subsection{The Original Matlab-based Script Did Not Include the Chi-square Test Analysis that was} featured in Radin et al. (2019)

In the following, it will be shown - by way of the historical research record - that the original Matlab-based script, which was used for the "pre-planned analysis" on March 11, 2013, did not include the chi-square test analysis as claimed now in Radin et al. (2019). Therefore, again, the claimed "true-positive result" cannot be part of the "pre-planned analysis". Briefly, Radin et al. (2019) argued that the so-called "true-positive result" is a function of a "pre-planned analysis" 
because - as Radin et al. (2019) have written - they have used "the script they provide" (see Sect. 3.4), i.e., the original Matlab script that was "available upon request" from Walleczek and von Stillfried (2019). However, was the Matlab script for the chi-square test analysis identical to the programming for the "pre-planned" variance analysis on March 11, 2013? The answer is no.

For explanation, as part of the independent analysis by Walleczek and von Stillfried (2019) of the results of the commissioned replication study (see Sect. 2.5), a request was made of Radin by the funder to provide a digital copy of the original Matlab script, i.e., the script that was used to analyze the data on March 11, 2013. Finally, in November 2016, a Matlab script was kindly provided by Radin to Walleczek and von Stillfried with the comment: "Here are scripts that will analyze the raw DS data in the way that I initially took." (Radin, personal communication, November 16, 2016). It was that script, provided by Radin in November 2016, that was used exclusively in the calculation of the mean differences as reported in Walleczek and von Stillfried (2019). Compare also Fig. 1B.

To confirm the validity of the received script, a simple test was made comparing the original mean differences as first viewed on March 11, 2013, at the IONS, and the independently calculated mean differences as obtained at Phenoscience Laboratories (Berlin). And indeed, the comparison of (i) the mean differences as were calculated with this script at Phenoscience Laboratories in 2016 (see. Sects. 7.2. and 7.3) and (ii) the original results for these same mean differences, i.e., "mean-shift magnitude" in Radin (2013), which were unblinded on March 11, 2013 (see Fig. 1B; mean shift), found good agreement for all eight planned tests (see also Figs. 3 and 4). This was reported in Walleczek and von Stillfried (2019). Note that the slight differences in the values for the individual means were attributed to the nonparametric bootstrap method involving a 300-loop random permutation process, as was explained in the legend to Fig. 4 in Walleczek and von Stillfried (2019).

However, in contrast to the mean differences as reported by Walleczek and von Stillfried (2019), the results of the chi-square test analysis as described in Radin et al. (2019) are inconsistent upon comparison with the original results. Again, a comparison (see Figs. $1 \mathrm{~A}$ and $1 \mathrm{~B}$ ) finds no agreement between (i) the results of the original variance analysis (Radin, 2013), as unblinded on March 11, 2013 (see Fig. 1B; variance shift) and (ii) the recent results of the chi-square test analysis in Table 1 of Radin et al. (2019), which is reported in Fig. 1A. For details see Sect. 3.1.

Consequently, the programming of the Matlab script which was forwarded by Radin to Phenoscience Laboratories in 2016 - although reporting near-identical results for the meandifference analysis (Fig. 1B; mean shift) - reported wholly different results when compared to the programming that was used for any of the variance analyses as performed on March 11, 2013 (Fig. $1 \mathrm{~B}$; variance shift). It must be concluded that a substantial change in programming had occurred - a change that was neither approved by, nor even mentioned to, the funder - sometime between March 2013 and November 2016. In summary, again, contrary to the claim by Radin et al. (2019), the so-called "genuine true-positive result" is not part of any "pre-planned analysis" but is a function of a post-hoc developed statistical analysis, i.e., of an unauthorized post-hoc alteration of the analytic routines sometime after March 11, 2013.

Note that it has proven to be relatively easy in the Radin DS-experiment to generate apparently "positive" effects - even in the absence of test subjects focusing on the DS-apparatus when using post-hoc developed, i.e., unplanned, statistical analyses (for details see Sects. 8). Crucially, this observation could be exploited for the purposes of HARKing in the effort to produce a desired "outcome" for the Radin DS-experiment. For an overview of planned and unplanned hypothesis testing see the table next. 
Table: Overview of planned and unplanned hypotheses. Planned hypotheses are those whose results were analyzed and unblinded with the decryption key provided by Walleczek on March 11, 2013. Planned hypotheses testing used either (i) the single-testing strategy which had been pre-specified in the original research proposal (see Sect. 2) or (ii) a multiple-testing strategy which had not been pre-specified in that proposal (see Sect. 3.3). An unplanned hypothesis is one that was developed post-hoc and whose results were calculated after unblinding the original results with the decryption key on March 11, 2013, i.e., in the absence of encryption and the blinding strategy (see Sect. 3).

\begin{tabular}{|c|c|c|}
\hline Type of Testing & $\frac{\text { Planned Hypotheses }}{\text { (March 11, 2013) }}$ & $\frac{\text { Unplanned Hypothesis }}{\text { (after March 11, 2013) }}$ \\
\hline $\begin{array}{l}\text { Single Testing } \\
\text { Pre-specified in } \\
\text { Radin (2011) }\end{array}$ & $\begin{array}{l}\text { Walleczek and von Stillfried (2019) } \\
\text { One outcome measure was used in } \\
\text { the confirmatory replication study }\end{array}$ & \\
\hline $\begin{array}{l}\text { Multiple Testing } \\
\text { Not Pre-specified } \\
\text { in Radin (2011) }\end{array}$ & $\begin{array}{l}\text { Unpublished negative results } \\
\text { (Radin, 2013) } \\
\text { Three new outcome measures } \\
\text { were added later - but still before } \\
\text { the data were unblinded (hence, } \\
\text { planned) - which were not part of } \\
\text { the confirmatory replication } \\
\text { study. }\end{array}$ & $\begin{array}{l}\text { Radin et al. }(2019,2020) \text { : } \\
\text { Did not disclose that the claimed } \\
\text { "true-positive outcome" derives } \\
\text { - in truth - from unplanned } \\
\text { hypothesis testing (in the form } \\
\text { of a chi-square test analysis). } \\
\text { Did not disclose that two } \\
\text { planned outcome measures based } \\
\text { on a variance analysis found a } \\
\text { negative result only } \\
\text { (Radin, 2013). }\end{array}$ \\
\hline
\end{tabular}

Next, consult Sect. 3.6, for the invalid reasoning behind the abandonment of the planned, directional hypothesis in favor of a novel, non-directional hypothesis to be analyzed by a chi-square test.

\subsection{Invalid Reasoning Behind the Decision to Abandon the Directional Hypothesis}

For completeness, it will be shown next that - in addition to the above concerns - the reasoning is not valid that was offered by Radin et al. (2019) for abandoning the pre-specified directional hypothesis, in favor of a non-directional, variance-type hypothesis. As will be explained below, one major design goal of the AMP-based research design was to fully control for potential time-dependent, biasing effects with the STC-experiment (O/X). However, Radin et al. (2019) falsely suggested that effective control experiments had not been put in place with the AMP when they argued that:

"Because the epoch-pairs were presented in random sequences, it was possible that as part of a session a participant could be presented with the instructions: OX XXXO. This sequence would require maintaining focused concentration for two minutes, with just a few short breaks. Given the 
difficulty in maintaining one-pointed attention for that length of time, we were not optimistic that the directional hypothesis would be supported. But under the secondary, non-directional hypothesis, differences in epoch-pair variance might still be detectable." (page 10)

To repeat, Radin et al. (2019) claimed that a significant directional effect was likely absent, because of the presumed "difficulty in maintaining one-pointed attention". However, for the 1,250 individual tests with the time-reversed $(\mathrm{O} / \mathrm{X}$ ) epoch pairs (STC), there was always a full 30 -sec resting (relaxed) period $(O)$ prior to performing the 30 -sec attention period $(X)$. Nevertheless, despite the systematic availability of resting periods $(\mathrm{O})$ prior to "focused attention" $(\mathrm{X})$, only a null result was observed with STC-epoch pair $\mathrm{O} / \mathrm{X}(\mathrm{N}=1,250$ tests), just like for the standard epoch pair $\mathrm{X} / \mathrm{O}(\mathrm{N}=$ $1,250)$. Compare the predictions versus the actual results for test categories $X / O$ and $O / X$ in Figs. $3 \mathrm{~A}$ and $3 \mathrm{~B}$ in Sect. 7.2. Therefore, the suggestion by Radin et al. (2019) that the "difficulty in maintaining one-pointed attention" was responsible for the lack of a directional effect is plainly contradicted by the findings with the STC-experiment of the AMP.

Additionally, as the careful reader will have noted already, the above claim by Radin et al. (2019) that a directional effect is absent because of the "difficulty in maintaining one-pointed attention" is also contradicted by Radin's own prior evidence reported to the funder in September 2013 (Radin et al., 2013) which had claimed a significant directional effect (see Fig. 2 in Sect. 3.2). Again, this prior claim of a directional effect, and the reasons for not publishing this directional effect (see Sect. 3.2), remain undisclosed in Radin et al. (2019, 2020).

Next, Sect. 4 will begin a review of the second major way in which Radin et al. $(2019,2020)$ have (i) deviated from the funder-approved AMP-based research design and (ii) misrepresented the research findings regarding the discovery of a false-positive effect in one of the predicted test categories in commissioned replication study (Walleczek and von Stillfried, 2019).

4. The Proposal to Correct for Multiple Testing by Radin et al. $(2019,2020)$ is Based on a Misrepresentation of the Single Testing Strategy that was Pre-specified in Radin (2011) and Used in Walleczek and von Stillfried (2019)

This particular misrepresentation by Radin et al. $(2019,2020)$ proceeds in two steps: First, they suggest that Walleczek and von Stillfried (2019) employed a strategy of "multiple testing", or "multiple comparison testing", for example, equal to the types of multiple testing described in Tukey (1991), Curran-Everett (2000), and Frane (2015). Second, they next suggest that, because of this purported strategy of "multiple testing", the results should have been adjusted by statistical correction for multiple testing, such as using a False Discovery Rate (FDR)-correction or Bonferronicorrection. After such an adjustment, the false-positive effect reported in Walleczek and von Stillfried (2019) would no longer be statistically significant. In the following, it will be shown that the first claim is incorrect and therefore the second step does not follow. Specifically, the suggestion by Radin et al. $(2019,2020)$ that a strategy of multiple comparison testing was used is in contradiction to the predictive single-testing strategy, using only the outcome predictions which were prespecified in the original research proposal by Radin (2011) and upon which the article by Walleczek and von Stillfried (2019) is based. In the context of this pre-specified analysis strategy, an adjustment for multiple (comparison) testing would be inappropriate. See Sect. $\underline{5}$ for a discussion of the multiple comparison problem as presented by Tukey (1991), Curran-Everett (2000), and Frane (2015), and a tutorial example for the correct statistical interpretation in the predictive single-testing strategy.

To begin, while it is true that several distinct pre-specified hypotheses were tested, each single test category (or hypothesis) was (i) associated with only one of three specific outcome predictions (null, positive, or negative, result), and (ii) tested using a separately-collected (single) 
data set. This represents not a single instance of random multiple testing but rather multiple instances of predictive single testing. In Walleczek and von Stillfried (2019), the standard definition of multiple testing was reviewed: The multiple-testing problem arises (i) whenever multiple hypotheses are tested using one and the same data set (or overlapping data sets), or (ii) whenever one and the same hypothesis is tested using multiple data sets; however, neither option (i) nor option (ii) was adopted in Walleczek and von Stillfried (2019). For the purpose of clarity, the distinction between multiple testing and single testing will be further explained next.

\subsection{What is Single Testing as Compared to Multiple Testing?}

The present work refers to the notion of 'single testing' as the testing of a given (single) null hypothesis using a single data set. A 'single data set' is the set of data that is collected uniquely for, and dedicated to, the testing of a specific prediction (null, positive, or negative) given a particular null hypothesis. Obviously, therefore, if a single-testing strategy is used in the (sequential) testing of multiple hypotheses, then each separate null hypothesis must be tested using its own dedicated data set. As will be explained in detail in Sects. 5 and 6, in the case of (predictive) single testing, no adjustment of $p$-values (Bonferroni, FDR or otherwise) is required; that is, no (random) multiple testing is performed.

Because some readers might be unfamiliar with the basic distinction of single testing versus multiple testing in the context of evaluating multiple hypotheses, the following paragraph by Rubin (2017) will be cited in full for illustrative purposes:

"Critics... might argue that researchers who conduct single tests of multiple hypotheses have a greater probability of incorrectly rejecting one of the null hypotheses that they test (i.e., making a Type I error). This is correct, but only insofar as those researchers also have a greater probability of incorrectly accepting, correctly accepting, and correctly rejecting one of their null hypotheses. These increased probabilities are due to testing more hypotheses rather than to any changes in the probabilities of correctly or incorrectly accepting or rejecting any individual hypothesis. To illustrate, consider a gambler who purchases 100 lottery tickets. Although this mass purchase increases the probability that the gambler will win the lottery, it does not increase or decrease the probability that any one of her lottery tickets will be the winning ticket. In the same way, a researcher who undertakes single tests of 100 different null hypotheses will have a relatively high probability of incorrectly rejecting one of those hypotheses, but she will not increase the probability of incorrectly rejecting each hypothesis." (page $6)$.

In contrast to single testing, 'multiple testing' refers either (i) to the random testing of multiple hypotheses using the same (single) data set or (ii) to the use of multiple data sets in the testing of a single null hypothesis. In the case of random multiple testing, statistical convention requires, of course, a (Bonferroni) correction or adjustment of the significance level for $p$-values. Again, however, the AMP-based research design does not involve random multiple testing, but represents a single-testing strategy based upon precise outcome predictions. To be sure, the fact that multiple testing (and without planned predictions) was not adopted in the commissioned replication study had already been clarified in the following paragraph in Walleczek and von Stillfried (2019):

"Regarding the statistical evaluation of each of the eight tested null hypotheses (see Table 1), the following explanation should be emphasized to avoid any misunderstanding: the AMP-based true- and sham- 
experiments employ fully dedicated, non-overlapping data sets exclusively to test each separate null hypothesis, i.e., $\mathrm{H}_{0}$-true- $\mathrm{X} / \mathrm{O}, \mathrm{H}_{0}$-true-O/O, $\mathrm{H}_{0^{-}}$ true- $\mathrm{X} / \mathrm{X}, \mathrm{H}_{0}$-true-O/X, $\mathrm{H}_{0}$-sham- $\mathrm{X}_{\mathrm{S}} / \mathrm{O}_{\mathrm{s}}, \mathrm{H}_{0}$-sham- $\mathrm{O}_{\mathrm{s}} / \mathrm{O}_{\mathrm{s}}, \mathrm{H}_{0}$-sham- $\mathrm{X}_{\mathrm{S}} / \mathrm{X}_{\mathrm{s}}$, and $\mathrm{H}_{0}$-sham- $\mathrm{O}_{s} / \mathrm{X}_{\mathrm{s}}$ (see Figure 1 and Table 1 ). Therefore, since (1) neither are used multiple, or overlapping, data sets in the test of one specific null hypothesis and (2) nor are multiple null hypotheses tested using one and the same, or an overlapping, data set, calculating any type of correction for multiple comparison testing, e.g., in the form of a Bonferroni correction, would be in error. That is, for this strictly predictive study design, no multiple comparisons that would necessitate a statistical (Bonferroni) correction were programmed in the Matlab script that was used to perform the preplanned, blinded statistical analysis (see Section "Materials and Methods")." (page 9).

Therefore, when Radin et al. $(2019,2020)$ suggested that the statistically-significant p-value in Walleczek and von Stillfried (2019) should be adjusted for multiple testing, they committed one of the following errors: (i) either they falsely assumed that the "eight planned tests" refer to the testing of one (universal) null hypothesis using eight data sets at random or (ii) they falsely assumed that the pre-specified research design involved the random testing of eight null hypotheses using only one data set; neither assumption (i) nor assumption (ii) are correct.

In summary, Radin et al. $(2019,2020)$ promoted the false narrative that the AMP adopts a non-predictive multiple-testing strategy, which it does not. Crucially, by falsely adjusting $p$-values (for "multiple comparison testing") when using a predictive single-testing strategy, there arises a two-fold risk: First, in the case of the standard experimental conditions (related to the trueexperiment in the AMP), the risk of missing a true-positive finding is increased, i.e., the falsenegative detection rate is increased (compare Sect. 7.2). Second, in the case of the shamexperimental conditions in the AMP (compare Sect. 7.3), the risk is increased of failing to reveal potentially hidden sources of false-positive effects in the experimental system, thereby increasing the risk of mistaking a false discovery for a true discovery with this system. Next, the important implications of the use of the AMP-based single-testing research strategy will be outlined (Sect. 3.2).

\subsection{Important Implications of the Predictive Single-Testing Research Strategy}

Given the above distinction between multiple testing and single testing, the statistical correction proposed by Radin et al. $(2019,2020)$ would only be adequate, for example, if the eight separate data sets would have been analyzed for the purpose of rejecting one and the same hypothesis. For example, if one would like to test the null hypothesis that false-positives occur at an above-chance rate in any one of the eight data sets at random, then that null hypothesis would not have to be rejected - obviously - based on the data collected in the commissioned study. According to the pre-specified research protocol (see Sect. 2), however, that was not the hypothesis to be tested.

In order to further clarify the approach represented by the AMP and to differentiate it from the multiple comparison problem that would require the statistical adjustment of $p$-values, the rationale behind the introduction of the AMP will be briefly recapitulated in the context of the metascience research reform movement. As was mentioned in the introduction, research strategies to counter the crucial problem of type- 1 error detection have received increased attention in recent years (e.g., see www.metascience.com). So far, one key advance has been the more widespread introduction of the strictly confirmatory research design which entails locking-in or pre-registering hypotheses and analytic methods in order to avoid manipulations, such as p-hacking and HARKing (Kerr, 1998). 
The AMP as adopted in 2011 as research strategy for the commissioned study conducted by Radin in 2012 and 2013 and as published by Walleczek and von Stillfried in 2019 not only implemented these confirmatory principles but, in addition, included (i) precise predictions and (ii) advanced procedures for helping to empirically detect false-positives which might be due to systematic experimental error or other artefacts. (This is why it is called an "advanced" methodology). Specifically, this means that not only were the different research hypotheses fully specified prior to the study, and the analysis protocol was locked-in, in form of a pre-written Matlab script which was applied to the data blindly (i.e. without knowing which data set pertains to which hypothesis nor whether it is an experimental or a control data set). In addition, the hypotheses to be tested included SNCs, SPCs, STCs, and the so-called sham-experiments, as detailed in Sect. 2. As a result, seven additional data sets were collected beyond the standard intervention experiment $(\mathrm{X} / \mathrm{O})$, with each data set having its own predicted measurement outcome.

To the untrained eye, this analysis of seven additional data sets might look like a "fishing expedition" (Gelman, 2014) in the sense of a multiple-testing problem, because, as Radin et al. (2019) correctly note, the probability of obtaining in any one of these data sets an effect with a pvalue smaller than a certain cut-off value increases proportionally with an increasing number of data sets, simply as a result of the inevitable statistical sampling error. What does not increase, however, is the probability by which a p-value smaller than a certain cut-off value is obtained in one specific data set where it is predicted to occur. No matter how many additional data sets are generated and analyzed, the probability that by chance alone a significant effect occurs through sampling error in exactly the data set or data sets where it was predicted to occur via pre-specified hypothesis is always adequately estimated by the unadjusted p-value or p-values. If this were not so, for example, the $p$-values for each scientific experiment would have to be adjusted as soon as another experiment is conducted.

One reason why this essentially quite simple reasoning may not be clear at first sight in the context of the commissioned study reported by Walleczek and von Stillfried (2019) is that here it could be wrongly assumed that some or all control conditions (SNCS, SPCS, STCs, and shamexperiments) actually test one and the same null hypothesis. This is, however, not the case. Instead, in full agreement with the funder, Radin (2011) had pre-specified that "This method is evaluated exclusively with epoch-pair differences because the aim is to test the effect of systematic fluctuations in the data (e.g., linear drifts or regular oscillations)" (see Sect. 2.3). This is the reason why it was pre-specified that for each experimental session "an identical session will be run as a sham control using the same epoch-pair orders used in the experimental session, except no one will be present." (see Sect. 2.3). As a result, each data set was collected under different test conditions (which is, by the way, why the different experimental and control groups were called either 'test categories' or 'test conditions' in Walleczek and von Stillfried, 2019). Thus, each separate data set was used to test a different null hypothesis, pertaining to exactly that specific test condition under which the data was collected (i.e., the specific epoch-pair order and the specific absence or presence of test subjects). Thus, for example, the sham-condition $\mathrm{X}_{\mathrm{s}} / \mathrm{O}_{\mathrm{s}}$ tested the hypothesis that the temporal sequence of epochs used in the experimental condition X/O could - by way of "systematic fluctuations" or correlated drifts in the data - create a statistically-significant (false-positive) effect. More precisely, what was tested with the data set $X_{s} / O_{s}$ was the null hypothesis that the specific epoch-pair order used in the standard experimental $\mathrm{X} / \mathrm{O}$, would - in the absence of subjects - not lead to an effect that could be mistaken for a true-positive effect, had it occurred in the presence of subjects. As a consequence, the results of the commissioned study can only be interpreted in a condition-specific and prediction-specific way.

For the above reasons, no more far-reaching conclusions were reported in Walleczek and von Stillfried (2019) than stating that (i) one false-positive effect was identified for one specific 
condition for which a false-positive effect was predicted to occur and (ii) the probability for this (false-positive) effect to happen by chance alone is adequately estimated by the unadjusted $p$-value of $p=0.02$ (see Walleczek and von Stillfried, 2019). Obviously, because of the prediction-specificity of the AMP-based analysis, no such conclusion would have been drawn, if the effect had occurred in the opposite (not predicted) direction, or in any of the other conditions. (In any such case the effect would not constitute a false-positive effect that could have been mistaken for a true positive effect if it had occurred in the corresponding experimental condition). In summary, from the pre-specified study protocol follows a distinct null hypothesis for each of the separate (single) data sets (for details see Table 1 in Walleczek and von Stillfried, 2019). What makes the data sets and the according hypotheses distinct is - among other things - the specific temporal sequence in which the epochs of $\mathrm{X}$ and $\mathrm{O}$ occur in the corresponding test session as well as the absence or presence of test subjects (for a more detailed explanation see the section called 'Advanced Meta-experimental Protocol' in Walleczek and von Stillfried, 2019).

In the attempt to support their proposal for adjustment of p-values due to "multiple comparison testing", Radin et al. (2019) refer to Frane (2015), and Radin et al. (2020) also to Tukey (1991) and Everett-Curran (2000). Therefore, in Sect. 5, these three articles will be briefly addressed, and it will be explained why they are not relevant to the case of the commissioned study conducted by Radin and reported by Walleczek and von Stillfried (2019). Thereafter, in Sect. 6, as an illustrative example, the statistical assumptions of a well-known parapsychological study will be reviewed. The review will demonstrate that it has been standard practice, even in parapsychology, to not adjust $p$ values in studies where multiple hypotheses are tested on separately-collected data sets, as long as a confirmatory and strictly predictive test strategy is adhered to. Again, in Sect. 7, the specific implementation of the confirmatory and strictly predictive research design in Walleczek and von Stillfried (2019) will be reviewed. Finally, Sects. 8 and 9 will present additional clarifications, and Sect. 10 will offer Conclusions and an Outlook.

\section{When Multiple Hypotheses are Tested on the Same Data Set, Even Planned Tests Require} Multiplicity Adjustment (Frane, 2015), and the Philosophy of Multiple Comparison Testing (Curran-Everett, 2000; Tukey, 1991)

The article by Frane (2015) is, among other things, concerned with the fallacy of mistaking "planned" hypothesis testing for a form of hypothesis testing that never requires statistical adjustments, or corrections. For example, Frane (2015) explained that:

“... many in the applied sciences consider it appropriate not to adjust for multiplicity if the tests were planned (i.e., if the hypotheses were specified a priori, meaning before the study began). In fact, researchers have frequently defended their unadjusted tests explicitly on the basis that the tests were planned." (Section 2)

However, the reason for not adjusting the tests in Walleczek and von Stillfried (2019) is not that they were 'planned' but that they did neither relate to the same data set, or overlapping data sets, nor did they test the same or overlapping hypotheses on one and the same data set (see Sect. 4). Furthermore, the above criticism by Frane (2015) does not distinguish between predictive and non-predictive, i.e., random, testing. Thus, the planned and predictive single-testing scenario employed in Walleczek and von Stillfried (2019) does not equal the planned and non-predictive multiple-testing scenario that was addressed by Frane (2015).

As an aside, the problems surrounding the issue of unplanned multiple testing may run even deeper than was suggested by Frane (2015). In the article, the impression is created that the problem of alpha-error accumulation can be accounted for by the appropriate correction both in cases of unplanned as well as planned multiple testing. This is, however, not necessarily so. 
Unplanned multiple testing obviously usually occurs post-hoc, i.e. after the data is known. Thus, the unplanned choice of a certain test will often not be random but based on the outcome found in a previous analysis of the same data set. In such a situation, the available tools to correct for multiple testing by estimating the so-called family-wise alpha error are no longer applicable, because these corrections assume that the choice of tests does not depend on outcomes of other chosen tests.

As another aside, a paragraph in Frane (2015) makes this recommendation for the researcher who is serious about avoiding a type- 1 detection error:

"When discussing how statistical procedures should be applied, emphasize the fundamental goals of those procedures. For example, the purpose of null hypothesis testing is to limit the rate at which scientific harm is caused by false discoveries, and the purpose of multiplicity adjustments is to preserve that limit when there are multiple simultaneous opportunities for scientific harm." (page 11)

As pointed out by Frane (2015), much scientific harm may result from false discoveries. Given that the confirmatory AMP-based research design used in Walleczek and von Stillfried (2019) was designed specifically to prevent false discoveries, this innovative approach should be welcomed by anyone who agrees with the valid concerns of Frane (2015). Again, the AMP represents an advanced methodology for empirically detecting, and quantifying, uncontrolled false-positive effects, which otherwise - might remain hidden and lead to false-positive conclusions (Walleczek and von Stillfried, 2019). If anything, therefore, a more sensitive, instead of conservative, statistical analysis might be applied, when the research goal is to minimize the risk of uncontrolled false-positive effects with the sham-experiment (see Sect. 4). Nevertheless, the same statistical standards were applied for all eight planned test categories, which includes the four sham-test categories (Walleczek and von Stillfried, 2019). See Sect. 7.3 for an explanation of a key tenet of the AMP-based test strategy which mandates that the statistical analysis of the sham-experiment must precisely duplicate that of the true-experiment.

Furthermore, Radin et al. (2020) have also referred to Tukey (1991) and Curran-Everett (2000) for the purpose of suggesting that the research design implemented in Walleczek and von Stillfried (2019) requires a correction for multiple comparison testing. Again, consult Sect. 4 which had already explained that a correction for (non-predictive) multiple comparison testing would be a mistake given that this form of non-predictive testing was not adopted as a research strategy. Next, the scenario for multiple-comparison testing by Curran-Everett (2000) will be briefly discussed and be shown to address a problem that is not relevant in the present case.

Briefly, Curran-Everett (2000) describes the standard multiple comparison problem, which is based on the well-known strategy of comparing multiple population means with each other for the purpose of identifying statistically-significant correlations among any of the collected population means at random. In that case, a statistical (Bonferroni) correction must be applied - of course - to account for the total number of collected population means. By contrast, in the case of the commissioned replication study, the pre-specified research strategy did not rely on any random comparisons among the 16 collected population means for the eight different test categories (see Sects. 2 and 4 for details); instead, comparisons were always limited to only two respective population means, e.g., $\mathrm{X}$ and $\mathrm{O}$, in the case of the specific test category $\mathrm{X} / \mathrm{O}$. It was never planned that a given population mean in one test category would be statistically compared - at random against one or more population means from any of the other test categories, such as those making up, e.g., epoch pairs O/X, X/X, or O/O. Indeed, Radin (2011) himself had unmistakably pre-specified what the appropriate comparisons and outcome predictions would be (compare Sect. 2): 
"The hypothesis is that comparisons between epochs with $\mathrm{X}-\mathrm{O}$ or $\mathrm{O}-\mathrm{X}$ comparisons should show a genuine difference, but $X-X$ and $0-O$ comparisons should show no difference." (page 4)

In summary, the test strategy used in the commissioned replication study was not the one discussed in Curran-Everett (2000), and therefore this criticism by Radin et al. (2020) violates the funder-approved original methodology which was strictly predictive and not based on multiple comparison testing along the lines of Curran-Everett (2000).

Equally, and finally, the seminal work by Tukey (1991), which has also been referred to in the recent commentary by Radin et al. (2020), describes an experimental scenario that does not equal the predictive scenario encountered in Walleczek and von Stillfried (2019). Like Curran-Everett (2000), Tukey (1991) described the multiple comparison problem as the problem of statistical correlations among multiple samples in a random, i.e., non-predicted, manner. To be sure, Tukey (1991) makes this abundantly clear from the outset when he describes the basic challenge of multiple comparison testing with the image of a card player:

"A man or woman who sits and deals out a deck of cards repeatedly will eventually get a very unusual set of hands. A report of unusualness would be taken quite differently if we knew it was the only deal ever made, or one of thousand deals, or one of a million deals, etc." (page 103)

Tukey (1991) uses the image of the card player to raise the issue of (i) "data dredging" (page 103 ) in multiple comparison testing and (ii) how to avoid false-positives when randomly comparing multiple samples against each other. Note also the prior image that was offered by Rubin (2017) of the gambler who purchases 100 lottery tickets in Sect. 4.1. For a detailed technical description and analytic examples consult Tukey (1991). Concluding, like Curran-Everett (2000), Tukey (1991) also does not suggest - contrary to the claim by Radin et al. $(2019,2020)$ - that a predictive test strategy requires the same statistical adjustments as a random test strategy.

\subsection{Tutorial Example: Statistical Interpretation in the Predictive Single-Testing Strategy}

As an illustration of the statistical interpretation in the AMP, imagine the following extreme scenario for tutorial purposes only: A new drug for decreasing pain is tested in the form of a pill (X). A total of 100 experimental tests are carried out, whereby - for each individual test - a separate data set is collected. Of the 100 tests, only one particular experiment, let's say test No. 61, does involve the test of verum pills $(X)$, which contain the active ingredient, against placebo pills $(0)$, i.e., only test No. 61 represents the standard experiment X/O. By contrast, all the other 99 planned tests do involve placebo pills (O) only as in the SNC-experiment (O/O; see Sect. 2.2). The pre-specified predictions for this 100-test experiment are, then, the following: only for test No. 61 is a truepositive effect predicted to occur, whereas none of the other 99 tests (with the control pills) are predicted to reveal true-positive effects. What is the appropriate threshold for achieving statistical significance for the single data set collected for X/O-test No. 61?

By the logic that is suggested by Radin et al. $(2019,2020)$, a logic that would ignore (i) the strictly predictive nature of the research design as well as (ii) the single-testing aspect of the AMPbased approach, that threshold should be set at $p \leq 0.0005$. For explanation, since (i) a total of 100 tests are performed and (ii) assuming a standard significance level of $p \leq 0.05$, after correction for 100 tests $(0.05 / 100)$, the calculated value would be $p \leq 0.0005$. However, again, the cut-off value at $p \leq 0.0005$ would only be the correct one to use if the design of the study were non-predictive, i.e., that of a 'fishing expedition' looking for effects at random - for any one of the 100 tests - without the predictive single-testing strategy. However, such a random-testing approach - without 
specifying test predictions - is neither the research design of the above experimental scenario with pills nor of the confirmatory replication study as was described in Walleczek and von Stillfried (2019). To repeat, both, the thought experiment with the pills as well as the commissioned Radin DS-experiment implement strictly predictive research designs using single-testing strategies (for the true-positive effect). For example, therefore, the correct threshold value for test No. 61, in the case of the above thought experiment with 100 single tests, is $p \leq 0.05$ (and not $p \leq 0.0005$ ).

In summary, it does not matter whether test No. 61 is part of an experiment that covers 100 , 1,000 , or 10,000 single tests. The cut-off value for statistical significance remains at $p \leq 0.05$ for the predicted outcome in X/O-test No. 61 - even if another 9,999 O/O-tests, i.e., SNC-experiments, are performed. Radin et al. (2020) are therefore misguided in citing Tukey (1991), Curran-Everett (2000), and Frane (2015), for the purpose of questioning the statistical interpretation that was used in the predictive single-testing regime of the AMP-based commissioned replication study (Walleczek and von Stillfried, 2019). To further illustrate this important point, next an independent research paradigm will be reviewed, which is familiar to all parapsychological researchers, i.e., the so-called Bem-experiment on "precognition" (Bem, 2011). This brief review of the Bem-experiment will confirm that it has been standard practice to not adjust, or correct, $p$ - values in situations where several hypotheses are tested in a strictly predictive manner, as long as the hypotheses are prespecified and tested on single, non-overlapping data sets.

\section{No Statistical Adjustments in Predictive Confirmatory Testing: The Case of Bem (2011)}

One of the decade's best-known parapsychological (psi) study is the Bem-experiment (Bem, 2011). The experiment is reviewed briefly in order to shed light on the practice of statistical adjustments, e.g., (Bonferroni) corrections, in the evaluation of possible effects (including of psi effects) when multiple hypotheses are evaluated using a predictive (single-testing) strategy (compare Sect. 4).

It will become apparent that there is agreement between skeptics and proponents regarding the proper statistical analysis of a predicted measurement outcome. Specifically, the review will show that an adjustment, or (Bonferroni) correction, is not required if (i) evaluating multiple hypotheses using dedicated (single) data sets, and (ii) adopting a confirmatory, strictly predictive, and condition-specific, test strategy.

This review concerns the statistical interpretation of data claiming the precognitive detection of erotic stimuli (Bem, 2011; Bem et al., 2011). Specifically, Bem (2011) claimed that a predictive (and confirmatory) strategy was employed in the experimental paradigm that involved the evaluation of five different hypotheses using five types of pictures (erotic, neutral, negative, positive, romantic). Bem predicted that the evaluation of only one of the five hypotheses, namely only the one testing the erotic pictures, would reveal an increase effect (as a function of test-subject precognition). Bem (2011) wrote the following statement of prediction: "Each session of the experiment included both erotic and nonerotic pictures randomly intermixed, and the main psi hypothesis was that participants would be able to identify the position of the hidden erotic picture significantly more often than chance (50\%)."

The key point is that Bem (2011) posited, and this was assumed also by Bem et al. (2011), that a predictive research design had been implemented, i.e., one predicting a psi effect both (i) for the specific test category (i.e., the erotic-pictures category only) as well as (ii) for the direction of the psi effect (i.e., an increase in hit rate only). Most importantly, no adjustment (Bonferroni-, FDR- or other correction), was performed in the Bem-experiment (for the five planned hypotheses), because again - the Bem-experiment had claimed a condition-specific prediction (Bem, 2011; Bem et al., 2011). Note, again, that if a Bonferroni correction were calculated for the original Bem-experiment (No. 1), then the effect for the erotic-pictures test category would lack statistical significance. 
Even the skeptical analysis of Wagenmakers et al. (2011) agrees with the position that a statistical (Bonferroni) correction is not required for the five planned hypotheses in Experiment No. 1 under the condition that the design of the Bem-experiment had indeed used pre-specified, condition-specific hypotheses and tested them using separate data sets. However, Wagenmakers et al. (2011) did express doubts about whether Bem et al. (2011) had - in fact - pre-specified their hypothesis that "... the main psi hypothesis was that participants would be able to identify the position of the hidden erotic picture significantly more often than chance $(50 \%)$." Having conducted their own review of the Bem-experiment (in particular of the critical Experiment No. 1 with the five hypotheses and upon finding no evidence for the pre-specification of the predicted measurement outcome regarding erotic stimuli), Wagenmakers et al. (2011) concluded that "... the foregoing suggests that strict confirmatory experiments were not conducted. This means that the reported $p$ values are incorrect and need to be adjusted upward", and this also "... means that Bem's Experiment 1 was to some extent a fishing expedition, an expedition that should have been explicitly reported and should have resulted in a correction of the reported $p$ value." It is clear from this brief review that the decisive step in the argument for, or against, a correction or adjustment of the $p$ value in Experiment No. 1, as reported in Bem (2011), is the decision regarding whether (i) a confirmatory (planned) research design had been implemented, (ii) the specific (predicted) measurement outcome had been pre-specified, and (iii) the specific hypotheses to be tested used distinct data sets.

Importantly, proponents (Bem et al., 2011) and skeptics (Wagenmakers et al., 2011) have agreed that if the above conditions $(i-i i i)$ are fulfilled, then there is no requirement for a correction or adjustment of the obtained p-value, even when multiple hypotheses are tested (e.g., five planned hypotheses in the case of Exp. No. 1 in Bem, 2011). In conclusion, the (planned) test strategy in Bem (2011) is thought - at least by the proponents - to represent a confirmatory and predictive design, and in that case - like the work in Walleczek and von Stillfried (2019) - does equally present a situation that is not addressed, e.g., by Frane (2015), as was discussed in Sect. 5.

Next, Sect. 7 will review key aspects and findings of the confirmatory and strictly predictive AMP-based test strategy, which was developed by Walleczek in 2011 using the above statistical standards, and was accepted by Radin (2011) for the FFF-sponsored replication study at the IONS (see also Sect. 2).

\section{The Confirmatory and Strictly Predictive Design in Walleczek and von Stillfried(2019)}

The above explanations as well as the review of prior research standards (see Sects. 4 to 6) illustrate the importance of correctly distinguishing between (random) multiple testing and (predictive) single testing in the AMP-based research design. Again, regarding the question (raised by Radin et al., 2019, 2020) of whether - or not - an adjustment for multiple testing is required for this particular replication attempt of the Radin DS-experiment, the correct answer depends on whether a predictive single-testing, or random multiple-testing, research design had been implemented in Walleczek and von Stillfried (2019).

As was described in Walleczek and von Stillfried (2019), the AMP-based research design represented a strictly predictive, single-testing regime, and it involved the use of a blinded (meta)protocol for data collection and analysis (see Sect. 2), with the decryption key for unblinding the data being in possession of Walleczek. In fact, the careful implementation of (i) the AMP-based testing strategy, (ii) the predictive design, and (iii) the blinding strategy, is not disputed by Radin et al. (2019). Importantly, in their manuscript, the authors confirm these three essential features of the commissioned replication study:

"The purpose of that experiment... was to test a "meta-protocol" designed by Walleczek." (page 7) 
"The proposed effect would be detected as a directional effect, i.e. as a decrease in the double-slit spectral component of the interference pattern, as compared to the same measure when attention was withdrawn."

(page 7 and 8)

"The randomly generated epoch-pair assignments were encrypted and stored in real-time. This ensured that the data could not be analyzed until all planned sessions were completed, at which time the decryption key would be provided by Walleczek." (page 15)

On March 11, 2013, the decryption key was provided by Walleczek to Radin, and the results were analyzed and unblinded for the evaluation of the eight null hypotheses as part of the planned single-testing replication design as pre-specified in Radin (2011). For details see Sect. 2, and for the planned variance analysis involving two additional outcome measures which were not, however, pre-specified in Radin (2011) consult Fig. 1B as well as Sect. 3.3, and the table in Sect. 3.5. For the eight planned single tests which had been pre-specified in Radin (2011) the eight statistical values were calculated for eight epoch-pair (mean) differences (see Sect. 2), using the pre-planned (and encrypted) statistical analysis as defined in the pre-programmed Matlab script. Note that this specific Matlab script, e.g., the one that was used for the confirmatory replication analysis of the eight planned single tests, has been referred to as the original Matlab script in Walleczek and von Stillfried (2019). Furthermore, as was reported in Walleczek and von Stillfried (2019), no multiple statistical comparisons across any of the eight epoch-pair differences were planned (see Sect. 2.2) in full agreement with standard statistical conventions (see Sects. 4 to 6). In fact, an adjustment or (Bonferroni) correction was not programmed in the original Matlab script.

Importantly, the results with the original Matlab script as reported in Walleczek and von Stillfried (2019), and as reproduced in Figs. 3 and 4 in the present report (see below), are in full agreement with the unblinded results from the original Matlab-based statistical analysis, which had been performed on March 11, 2013, in the physical presence of both Radin and Walleczek at the IONS. As was mentioned in Sect. 3, on March 11, 2013, Radin forwarded to Walleczek a digital copy of the original statistical findings as were revealed on that day, in the form of a power-point presentation (Radin, 2013). For these results see Fig. 1B. Importantly, the results for the measure called "mean-shift magnitude" in the forwarded presentation agree with those presented in Walleczek and von Stillfried (2019). Again, note that the slight differences in the values for the individual means were attributed, also by Radin (personal communication), to the nonparametric bootstrap method involving a 300-loop random permutation process, as was reported in the legend to Fig. 4 in Walleczek and von Stillfried (2019).

In summary, in full acceptance of the confirmatory and predictive AMP-based research design, Walleczek and von Stillfried (2019) used only the original Matlab script for the statistical analysis of the planned predictions for this replication study. Again, the predictive single-testing and confirmatory (planned) research design sought to minimize the risk of p-hacking and HARKing (Kerr, 1998).

\subsection{Changing the Form of Hypothesis Testing After the Results are Known (HARKing)}

The misrepresentation by Radin et al. $(2019,2020)$ of the confirmatory replication design in Walleczek and von Stillfried (2019) represents a type of HARKing (Kerr, 1998). That is, in their recent criticism, Radin et al. $(2019,2020)$ departed from the original Matlab script for confirmatory hypothesis testing - subsequent to breaking the blinding code and having examined the data on March 11, 2013. This violated the original protocol for planned hypothesis testing as detailed in Radin (2011). The funder-approved protocol unmistakably stated that the code for the final results was only to be broken after all the data had been collected and analyzed. See Sect. 2.2. for the 
statement by Radin (2011) which confirms that "The code will be broken only after all data from all 25 subjects (a total of 250 experimental and 250 sham sessions) has been collected and analyzed".

However, it is apparent that - for their recent criticism - Radin et al. $(2019,2020)$ have switched the analytic methodology - sometime after the original examination of the data on March 11, 2013 - from (i) the predictive single-testing strategy that was based upon the outcome predictions as pre-specified in Radin (2011), to (ii) a non-predictive multiple-testing strategy which (falsely) assumed that the funder-approved research hypotheses merely posited the random (nonpredicted) occurrence of effects, i.e., without discriminating between effect positions or directions. For the correct research record see Sects. 2.2 and 2.3. This undisclosed post-hoc change by Radin, which, again, was neither revealed by Radin et al. $(2019,2020)$ nor approved by the funder, is incomprehensible given that the pre-specified task of the commissioned replication study was to confirm the specific predictions (Sect. 2.2) concerning (i) “... the positive results observed in earlier experiments..." (Sect. 2.1), and (ii) the all-important question of whether the earlier results "... may have been due to systematic but previously undetected measurement errors" (see Sect. 2.1). In summary, for the confirmatory research design, all instances of non-disclosed post-hoc changes in the statistical routines for planned hypothesis testing represent instances of HARKing (Kerr, 1998).

\subsection{Confirmatory True-experiments in Walleczek and Von Stillfried (2019)}

The commissioned replication study reported in Walleczek and von Stillfried (2019) had implemented a confirmatory and predictive research design. It is evident also from the review in Sects. 4 to 6 that an adjustment of $p$-values is not required in the present case of an exclusively predictive research design involving the single-testing approach. For an illustration see Fig. 3 below, which provides a comparison between the effect predictions and the actual results which were obtained with the four test categories (or epoch pairs) in the presence of observer consciousness (i.e., the so-called "True-experiment"; see also Sect. 2.2)

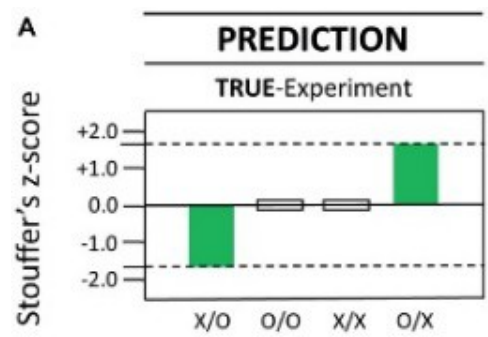

\begin{tabular}{|c|c|c|c|c|}
\hline Category & $\mathrm{X} / \mathrm{O}$ & $\mathrm{O} / \mathrm{O}$ & $x / x$ & $0 / x$ \\
\hline z-score & $\leq-1.64$ & $\begin{array}{l}<+1.96 \\
>-1.96\end{array}$ & $\begin{array}{l}<+1.96 \\
>-1.96\end{array}$ & $\geq+1.64$ \\
\hline
\end{tabular}

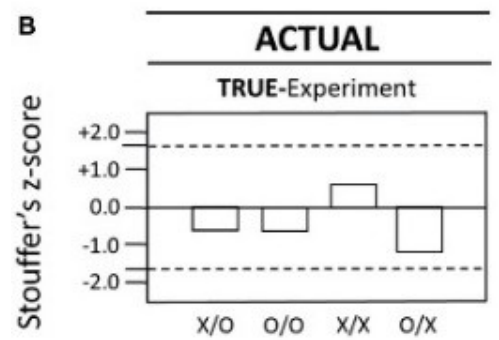

\begin{tabular}{|c|cccc|}
\hline Category & $X / O$ & $0 / 0$ & $X / X$ & $O / X$ \\
\hline z-score & -0.61 & -0.60 & +0.60 & -1.16 \\
SEM & \pm 0.0070 & \pm 0.0074 & \pm 0.0067 & \pm 0.0064 \\
\hline
\end{tabular}

Figure 3: True-experiment - With Observer Consciousness. For details see legend to Fig. 4 in Walleczek and von Stillfried (2019). For explanations of the four null hypotheses X/O, O/O, X/X, and $\mathrm{O} / \mathrm{X}$ see Table 1 in Walleczek and von Stillfried (2019). X = "attention"; $\mathrm{O}=$ "attention withdrawn".

The null results, as were observed with the epoch-pair differences for $\mathrm{X} / \mathrm{O}$ and $\mathrm{O} / \mathrm{X}$ in the true-experiment (Fig. 3B), could have been published on their own. That would have simply represented a negative replication attempt. However, an additional goal had been pre-specified for the commissioned study (see Walleczek and von Stillfried, 2019). That is, a minimum stress 
test for the used research methodology, including for the reliability of the used statistical analysis, was also performed - with the so-called "Sham-experiment" (see also Radin, 2011, in Sect. 2.3). For that purpose, the AMP always includes a test strategy that exactly duplicates all aspects of the statistical analysis from the true-experiment in the sham-experiment (Sect. 7.3).

\subsection{Counterfactual-Meta-experimentation: Sham-experiments Without the Test Variable}

The sham-experiments represent a minimum stress test for confirming the overall validity of the adopted (statistical) methodology in order "... to ensure that the experimental intervention (in our case the act of focusing attention towards the DS-system) has a real effect." (Radin, 2011; see Sect. 2.3). Again, a "real effect" was predicted only for epoch pairs $X / O$ and $O / X$ in the trueexperiment, and only for a decrease in $\mathrm{X} / \mathrm{O}$ and for an increase in $\mathrm{O} / \mathrm{X}$ (compare Fig. $4 \mathrm{~A}$ ).

As was already reviewed in Sect. 2.3, Radin (2011) had summarized the sham-experimental test strategy of the confirmatory AMP-based research design by Walleczek as follows: "The companion 'sham' protocol will be a control test consisting of the same protocol, but without subjects present." Specifically, after each (true)experimental session, “... an identical session will be run as a sham control using the same epoch-pair orders used in the experimental session, except no one will be present" (Radin, 2011).

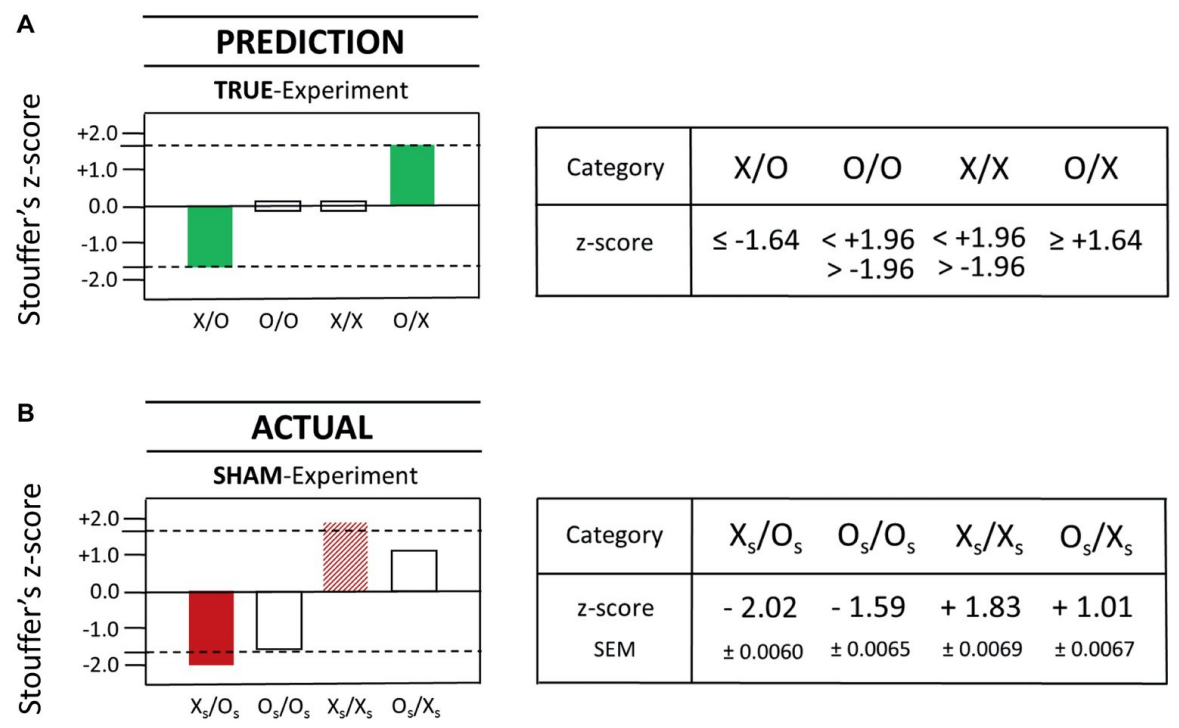

Figure 4: Sham-experiment - Without Observer Consciousness. For details see legend to Fig. 5 in Walleczek and von Stillfried (2019). For an explanation of the four null hypotheses $\mathrm{X}_{s} / \mathrm{O}_{s}, \mathrm{O}_{s} / \mathrm{O}_{s}$, $\mathrm{X}_{S} / \mathrm{X}_{\mathrm{S}}$, and $\mathrm{O}_{\mathrm{S}} / \mathrm{X}_{\mathrm{s}}$ see Table 1 in Walleczek and von Stillfried (2019).

The reasoning behind this advanced control-test strategy is the following: The used statistical analysis could only yield a credible positive result for the predicted epoch pair (e.g., for $\mathrm{X} / \mathrm{O}$ in Fig. $4 \mathrm{~A}$ ) if it could also be confirmed that the used analysis does - in fact - yield only a null result for the predicted epoch pair - upon removing the test variable from the experiment (e.g., for $\mathrm{X}_{\mathrm{S}} / \mathrm{O}_{\mathrm{s}}$ in Fig. 4B). This means that each one of the four different epoch pairs represent one of four distinct null hypotheses (see legend to Fig. 4). This control-test strategy is known as counterfactual meta-experimentation (CFME), and CFME seeks to replicate the true-experimental conditions - as closely as possible - in the absence of the (source of the) test variable (for details see Walleczek and von Stillfried, 2019). In statistical language, the goal of CFME is to accept a particular null hypothesis in the absence of the test variable $X$ - by way of demonstrating null results. CFME reduces the risk of mistaking a false discovery for a true discovery with a given null hypothesis. 
Importantly, the AMP-based research design mandates that the statistical analysis for the sham-experiment (see Fig. 4B) is to duplicate the statistical analysis used in the true-experiment (see Fig. 3B). To repeat, the statistical analysis in the sham-experiment must mirror exactly the statistical analysis of the true-experiment; otherwise, the whole point of performing the sham-experimental strategy - as a minimum stress test for the reliability of the true-experimental (statistical) performance - would be for naught. For example, given that a statistical correction for multiple testing is not required in the case of the four test categories in the true-experiment (see Fig. 3), such a correction must be absent from the sham-experiment also (compare Fig. 4).

For explaining the sham-experimental test strategy, again, the Bem-experiment in Sect. 6 can serve as a useful tutorial example. Imagine that experiment No. 1 with the five categories of hidden pictures were performed exactly as described in Bem (2011). However, this time, unbeknownst to both test subjects and the investigator, no pictures were "hidden" (behind the curtain) throughout the whole experimental series - from start to finish in accord with the CFMEstrategy. Then, obviously, if a statistically-significant effect would be claimed for the predicted category (with this type of sham-experiment), then that effect must count as a false-positive effect. The simple reason is that if there were no hidden pictures to detect by way of (psi) pre-cognition - in view of the main psi hypothesis that "... participants would be able to identify the position of the hidden erotic picture significantly more often than chance (50\%)" - then a statistically-significant increase effect (in hit rate) for the predicted category would necessarily represent a false-positive effect.

The same logic for the sham-experimental approach, as was explained for the Bemexperiment, was actually implemented and tested in the commissioned replication study of the Radin DS-experiment (Walleczek and von Stillfried, 2019). For comparison, in the hypothetical tutorial example with the Bem-experiment, the (removed) test variable $(X)$ would be the "hidden pictures", whereas in the Radin DS-experiment, $X=$ "observer consciousness" (e.g., seeking to decrease DS-light intensity by collapsing the wave function with observer "attention"; Fig. 4A).

These sham-experiments - without observer consciousness in the case of the Radin DSexperiment - provided a minimum stress test for the overall performance of the adopted research design. Specifically, with an independent set of (sham)experiments (i.e., 250 additional test sessions without test subjects), the data were recorded and statistically analyzed, again in a blinded fashion, for identically-randomized epoch-pair assignments (i.e., $\mathrm{X}_{s} / \mathrm{O}_{s}, \mathrm{O}_{s} / \mathrm{O}_{s}, \mathrm{X}_{\mathrm{s}} / \mathrm{X}_{\mathrm{s}}, \mathrm{O}_{s} / \mathrm{X}_{\mathrm{s}}$ ) as taken from the true-experiments $(\mathrm{X} / \mathrm{O}, \mathrm{O} / \mathrm{O}, \mathrm{X} / \mathrm{X}, \mathrm{O} / \mathrm{X})$; however, the pre-specified 5,000 test trials were collected in the absence of observer consciousness (compare with Radin, 2011, in Sects. 2.2 and 2.3).

For the sham-experiment (without observer consciousness), if an effect were identified for (i) a predicted test category (e.g., epoch pair $\mathrm{X}_{\mathrm{s}} / \mathrm{O}_{\mathrm{s}}$ ) and (ii) a predicted effect direction (a decrease), then this would indicate that the used methodology can generate the type of false-positive effect which could easily be mistaken for a true-positive effect by the investigator; this as a possible function of "... systematic fluctuations in the data (e.g., linear drifts or regular oscillations)", as was described in Sect. 2.3 (Radin, 2011). In fact, such an actual (false-positive) effect, which could be mistaken for the predicted "real effect" (see Sect. 2.3), was identified in the Radin DS-experiment (compare the prediction and the actual result to the left of Fig. 4 above). In summary, the falsepositive detection rate was determined to be $50 \%$ for the commissioned Radin DS-experiment. That is, one of the two predicted "real effects" was - falsely - replicated in the absence of test-subject observer consciousness, which indicated the presence of a significant amount of systematic class-A error in the performance of the Radin DS-experiment (for details see Walleczek and von Stillfried, 2019). 
Note, again, that Radin (2011) had confirmed that "... the aim is to test the effect of systematic fluctuations in the data (e.g., linear drifts or regular oscillations), and to ensure that the experimental intervention... has a real effect" (Sect. 2.3). For the exact replication of the temporal sequence of the randomly-generated epoch-pair assignments (i.e., $\mathrm{X}_{\mathrm{S}} / \mathrm{O}_{\mathrm{s}}, \mathrm{O}_{s} / \mathrm{O}_{\mathrm{S}}, \mathrm{X}_{\mathrm{S}} / \mathrm{X}_{\mathrm{s}}$, etc.) in the confirmatory CFME-based design see Walleczek and von Stillfried (2019).

Summarizing, a blinded, and predictive, test strategy was implemented in the commissioned replication study for both the true- and sham-experiments (i.e., for the 5,000 test trials each). The results and conclusions as reported in Walleczek and von Stillfried (2019) are entirely consistent with the confirmatory and strictly predictive research design, and for the technical details see that article. Next, Sect. 8 will discuss the relation of the findings in Walleczek and von Stillfried (2019) to other work following the publication of the original, FFF-(co)funded study (Radin et al., 2012).

\section{Three Research Groups Have Either Reported Null Results or Discovered False-positive Effects in the Radin DS-experiment \\ Together, the reports by three research groups in 2019 (other than by Radin and co-} workers) paint a worrisome picture for the Radin DS-experiment. That is, these reports after the publication of the original, FFF-(co)funded study (Radin et al., 2012), found the following: (i) two independent analyses found null results only, when using pre-specified statistical analyses, i.e., when HARKing was absent (Guerrer, 2019; Walleczek and von Stillfried, 2019), and (ii) three separate analyses discovered the presence of false-positive effects for control conditions (Tremblay, 2019; Walleczek and von Stillfried, 2019; von Stillfried and Walleczek, 2019). See Sects. 8.1 to 8.3.

\subsection{Guerrer (2019): Only Null Results are Reported with the Pre-registered Analysis}

Recently, Radin et al. (2020) have cited the data by Guerrer (2019) as evidence in support of their claims; however, the independent work by Guerrer (2019) reported a failure to replicate the prior claim by Radin et al. (2012) of an observer-dependent decrease effect on DS-light interference intensity: "The formal experiments, based on a directional hypothesis, were not statistically significant" (Guerrer, 2019). Only for a post-hoc introduced analysis for a bi-directional effect did Guerrer (2019) claim "...a 2.75 sigma outcome." In summary, the null results of the pre-registered (planned) analysis of Guerrer (2019) are in agreement with the null results found with the planned (i.e., encrypted) analysis of the commissioned study as reported in Walleczek and von Stillfried (2019).

\subsection{Tremblay (2019): False-positive Effects in the Control Group are Reported}

The report by Walleczek and von Stillfried (2019) of the false-positive effect in the Radin DSexperiment is consistent also with an independent reanalysis of the performance of the Radin DSexperiment by Tremblay (2019). He reported - for statistical analytic conditions showing apparent positive effects - that the Radin DS-experiment produced false-positive effects in the control group also. The conclusions by Tremblay (2019) had also been mentioned in Walleczek and von Stillfried (2019):
"Recent evidence from an independent re-analysis of sham-type control data from prior Radin DS-experiments supports this view also: Tremblay (2019) reported that 'part of the control data is also found anomalous,' and that this 'undermines the anomalies found in the human data, and weakens possible conclusions to be drawn from this data set."' (page 16).

Radin et al. $(2019,2020)$ chose to ignore, however, the report of these anomalous (falsepositive) results as discovered in the Radin DS-experiment by Tremblay (2019), and - inexplicably these false-positive effects were omitted from their discussion of prior results (Radin et al., 2019, 2020). See versions 1 to 4 of the pre-print by Radin et al. (2019), and Radin et al. (2020). 


\subsection{Von Stillfried and Walleczek (2019): Sham-experiments Reveal False-Positive Effects}

Von Stillfried and Walleczek (2019) empirically analyzed the statistical methods used by Radin and co-workers in three of their DS-experiments (for references see von Stillfried and Walleczek, 2019). Radin kindly provided a DS-apparatus and a Matlab-script for data-analysis using three analysis-parameters closely resembling the ones used in the respective studies. Applying the script to new data collected with the DS-apparatus under sham-conditions at Phenoscience Laboratories in Berlin (in the absence of any test subjects), it was found that (i) an erroneous dataprocessing procedure included in the script ("trimming") created false-positives and (ii) the different analysis-parameters differ so strongly that they cannot be considered replications of each other and were likely not pre-specified by Radin but developed post-hoc. Next, in Sects. 9 and 10, additional claims and criticisms by Radin et al. $(2019,2020)$ will be addressed and conclusions drawn.

\section{Criticisms by Radin et al. $(2019,2020)$ of the Funder}

Radin et al. (2019) wrote in the abstract: "The first author of the critique had funded the study and requested that it not be published." First, the first author did not offer a critique of a study. Instead, Walleczek and von Stillfried (2019) published the original findings of a replication study which (i) was commissioned by the FFF and Walleczek and (ii) had used the advanced methodology developed by Walleczek (see Sect. 2 for details). The agreement between the IONS and the FFF for performing the independent evaluation is also accounted for in Materials and Methods in Walleczek and von Stillfried (2019).

Second, what had been requested of Radin by the funder was to publish the results of the commissioned replication study in a way that honored the pre-specified confirmatory research design, but not any post-hoc findings based on the malpractice of (undisclosed) HARKing (see Sect. 3). In a regrettable new development in August 2020, Radin decided to add yet another false claim in version No. 5 of Radin et al. (2019). Incredibly, that false claim now suggested that it was the funder (Walleczek) who had requested of Radin to publish the results of a post-hoc analysis. For a direct refutation of this (false) claim, consult Sect. 3.2 which describes the (first) attempt by Radin to publish the results from a post-hoc analysis in 2013, an attempt that was - as the research record clearly demonstrates - rejected by the funder already in 2013. To be sure, Walleczek had never requested of Radin to falsify the research record by releasing the result of a (non-planned) post-hoc analysis (e.g., using a chi-square test or any other form of statistical analysis), and then to claim that - instead - that result derived from blinded (planned) hypothesis-testing as part of the original protocol; again, the original protocol did not include the chi-square test, i.e., the statistical test upon which Radin et al. $(2019,2020)$ had based their so-called "true-positive" result. Consult Fig. 1 for detailed documentation of the absence of the chi-square test as part of the original analysis as performed on March 11, 2013. Unfortunately, as was noted also in the recent commentary by Walleczek and von Stillfried $(2020)$, Radin et al. $(2019,2020)$ chose to leave undisclosed the fact that the chi-square test analysis was - in truth - a post-hoc analysis, and, therefore, the so-called "genuine true-positive" result is due to (undisclosed) HARKing (compare Fig. 1). This regretful behavior would have severely compromised the scientific integrity of the commissioned replication study (see Sects. 2 and 3 as well as Table 1 for the actual research record). Such behavior by Radin was the main reason for why an independent analysis of the funder-commissioned replication study had to be published to keep intact the project's integrity (Walleczek and von Stillfried, 2019, 2020).

Third, Walleczek, who - again - is director of the FFF, i.e., the sponsoring agency that had both funded the commissioned replication study at the IONS as well as (co)funded the original study (Radin et al., 2012), had great interest to find out whether (i) the original, FFF-(co)funded study was reproducible, and (ii) a false-positive effect could be ruled out as a probable explanation of the original results reported in Radin et al. (2012). 


\section{Conclusions and Outlook}

The present work has clarified that the misrepresentations by Radin et al. $(2019,2020)-$ of the results and advanced protocol in Walleczek and von Stillfried (2019) - are entirely based on the detrimental practice of (undisclosed) HARKing that is used by Radin et al. $(2019,2020)$. The malpractice of HARKing by Radin is also the topic of the recent commentary by Walleczek and von Stillfried (2020) with a focus on two specific points: First, the so-called "true-positive outcome" was - contrary to the claim by Radin et al. $(2019,2020)$ - not the result of planned hypothesis testing, but was developed post-hoc, i.e., after unblinding the original results (see Fig. 1 in Sect. 3.1). Crucially, it has proven to be relatively easy to generate apparently "positive" effects in the Radin DS-experiment upon using post-hoc developed, i.e., unplanned, statistical analyses, which is an observation that could be exploited for the purposes of HARKing in the attempt to obtain a desired "outcome" (see also Sects. 8 and 9). Second, Radin et al. $(2019,2020)$ falsely suggested that the newly developed protocol, i.e., the AMP, implements a random, non-predictive test strategy when in fact - the AMP-based test strategy is strictly predictive. It is remarkable that Radin et al. (2019, 2020) have chosen to ignore this obvious design feature of the commissioned replication study given that the terms 'prediction' or 'predicted' show up more than 50 times in the article by Walleczek and von Stillfried (2019), including in the results figures which prominently illustrate each of the distinct test predictions (see Figs. $3 \mathrm{~A}$ and $4 \mathrm{~A}$ in the present manuscript). This amounts to yet another case of HARKing because Radin et al. $(2019,2020)$ abandoned (i) the planned predictive single-testing strategy and - without disclosing this fact - switched to (ii) an unplanned random multiple-testing strategy - subsequent to breaking the blinding code (see Sect. 7.1).

Briefly, Radin et al. (2020) sought to misrepresent the pre-specified research design by falsely suggesting - again not even mentioning the test predictions - that "... the experiment involved eight comparisons performed on non-overlapping data partitioned from a single dataset". For explanation, Radin et al. (2020) are promoting the false (post-hoc) narrative that the eight different hypotheses merely posited the random occurrence of (arbitrary) effects for "a single dataset". However, in truth, the pre-specified testing strategy was not random but it was strictly predictive, and for the correct research record see the original statement of prediction by Radin (2011) in Sect. 2. Therefore, any statistical corrections for non-predictive, i.e., random, multiple testing would thus violate the AMP-based test strategy which implemented the planned outcome predictions as pre-specified by Radin (2011): True-positive effects were predicted for only two $(12.5 \%)$ of the 16 possible measurement outcomes of the eight planned single-test categories. That crucial fact is entirely ignored by the (randomizing) post-hoc analysis which is promoted by Radin et al. $(2019,2020)$ using HARKing (Sect. 7.1). For a tutorial example of the statistical interpretation in the predictive single-testing strategy as implemented in the AMP see Sect. 5.1.

Summarizing, contrary to the suggestion by Radin et al. $(2019,2020)$, this commissioned replication study was not designed as a 'fishing expedition' for false-positive effects, i.e., for effects to be observed at random for any one of the "eight planned tests" - for any arbitrary effect direction or position (see Sect. 2 for documentation). By contrast, the planned AMP-based research design was strictly predictive both in terms of the position and direction of the effect (see Figs. 3 and 4), and, hence, the statistically-significant, false-positive effect in the Radin DS-experiment persists (Walleczek and von Stillfried, 2019). In particular, using a counterfactual strategy of metaexperimentation (i.e., of strictly predictive sham-experiments in the absence of observer consciousness; see Sect. 7.3), it was determined that "... the false-positive effect, which would be indistinguishable from the predicted true-positive effect, was significant at $p=0.021(\sigma=-2.02 ; \mathrm{N}=$ 1,250 test trials)". Compare the prediction and the actual result to the left of Fig. 4 , and for details see Walleczek and von Stillfried (2019). In short, the Radin DS-experiment has failed the minimum stress test in the commissioned study, and the discovered false-positive effect is consistent with an 
independent reanalysis of the Radin DS-experiment by Tremblay (2019), who found - for analytic conditions showing apparent positive effects - that the Radin DS-experiment produced false-positive effects in the control group also (see Sect. 8).

By referring to the work of Frane (2015), Radin et al. $(2019,2020)$ have drawn attention to the challenge of how to best "... limit the rate at which scientific harm is caused by false discoveries" (see Sect. 5). The metascience reform movement (see www.metascience.com) has identified the shift from exploratory to confirmatory research strategies as an effective tool for avoiding false discoveries, and Walleczek and von Stillfried (2019) had concluded their recent article as follows:

"For the future, our recommendation is for researchers to discontinue the exploratory research approach toward the Radin DS-experiment (see Table 3) and to start implementing the methods and protocols that are consistent with strictly confirmatory research practices and designs (e.g., Simmons et al., 2011; Wagenmakers et al., 2012; Munafò et al., 2017; von Stillfried and Walleczek, in preparation); only confirmatory research designs might put to rest the long-standing questions about the possibility of type- 1 error as part of any conceptual replication attempt relating to the purported consciousness effect." (page 17)

The shift from exploratory to confirmatory research practices would substantially lower the risk of false discoveries in the Radin DS-experiment, including in future replication experiments. However, in their recent call for additional replications, Radin et al. (2019) have failed to strongly recommend - for replications of the Radin DS-experiment - the adoption of wholly confirmatory research practices, like those that were adopted in the replication report by Walleczek and von Stillfried (2019). Most importantly, given that the specificity of the Radin DS-experiment for detecting 'observer consciousness' remains unconfirmed, how to tell whether any of the previouslyreported positive results are either true-positive or false-positive? Radin et al. $(2019,2020)$ have failed to mention a credible control mechanism that would be capable of distinguishing a (potential) true-positive observer effect from a false-positive observer effect. Therefore, short of radical improvements in the research standards for the Radin DS-experiment (see Walleczek and von Stillfried, 2019), it is likely that false discoveries will continue to be promulgated, including for replication experiments. Put simply, the possibility of a true discovery depends entirely on credible, empirical proof for the absence of false-positive effects in this experiment.

Finally, both the results reported in Walleczek and von Stillfried (2019) as well as the review of recent findings in Sect. 8 indicate the urgent need to improve research standards, because evidence is mounting that the Radin DS-experiment is prone to producing false discoveries. For example, von Stillfried and Walleczek (2019) have shown that even small post-hoc adjustments in the setting of analytic parameters for an exploratory statistical analysis (e.g., HARKing) could easily generate false-positive conclusions in the Radin DS-experiment (see Sect. 8.3). The present authors call for the implementation of advanced control-test strategies, like the confirmatory AMP-based research design (Walleczek and von Stillfried, 2019), for empirically detecting and preventing uncontrolled false-positive effects in the Radin DS-experiment.

\section{Author Contributions}

JW and NvS jointly wrote this article and have full agreement regarding its content.

\section{Conflict of Interest Statement}

The authors declare that the research was conducted in the absence of any commercial or financial relationships that could be construed as a potential conflict of interest. 
Acknowledgments: The work by JW and NvS at Phenoscience Laboratories

(www.phenoscience.com) was supported in large part by the Fetzer Franklin Fund of the John E. Fetzer Memorial Trust, as was the commissioned replication study at the Institute of Noetic Sciences.

\section{$\underline{\text { References }}$}

Bem, D. J. (2011) Feeling the Future: Experimental Evidence for Anomalous Retroactive Influences on Cognition and Affect. J. Pers. Soc. Psychol. 100, 407-425. DOI: 10.1037/a0021524

Bem, D. J., Utts, J., and Johnson, W. O. (2011) Must Psychologists Change the Way They Analyze Their Data? J. Pers. Soc. Psychol. 101, 716 - 719.

Curran-Everett, D. (2000) Multiple Comparisons: Philosophies and Illustrations. Am. J. Physiol. Regulatory Integrative Comp. Physiol. 279, R1 - R8, 2000. http://www.ajpregu.org 0363-6119/00.

Gelman, A. and Loken, E. (2014). The statistical crisis in science: data-dependent analysis - a" garden of forking paths" - explains why many statistically significant comparisons don't hold up. American Scientist, 102(6), 460-466.

Guerrer, G. (2019). Consciousness-related Interactions in a Double-slit Optical System. https://osf.io/qdkvx

Frane, A. V. (2015) Planned Hypothesis Tests are not Necessarily Exempt from Multiplicity Adjustment. J. Res. Practice 11, P2.

Kerr, N. L. (1998) HARKing: Hypothesizing After the Results are Known. Pers. Soc. Psychol. Rev. 2: 196-217. https://doi.org/10.1207/s15327957pspr0203 4

Radin, D. (2011) Research Proposal to the Fetzer Franklin Fund of the John E. Fetzer Memorial Trust; Institute of Noetic Sciences, Consciousness Research Laboratory; October 5, 2011.

Radin, D. (2013) Power-Point Presentation with Four Slides of The Statistical Results After Breaking the Blinding Code for the Commissioned Replication Study of the Radin Double-slit Experiment. Personal Communication of a Digital Copy of the Presentation; March 11, 2013

Radin, D., Michel, L., Galdamez, P., Wendland, R., Rickenbach, R., and Delorme, A. (2012). Consciousness and the Double-slit Interference Pattern: Six Experiments. Phys. Essays 25, 157-171. DOI: 10.4006/0836-1398-25.2.157

Radin, D., Michel, L., and Delorme, A. (2013) Consciousness and the Double-slit Interference Pattern: Experimental Investigation of a Force-like Model. Report on the IONS Fetzer-Franklin Double-slit Experiment, v5. September 14, 2013.

Radin, D., Wahbeh, H., Michel, L., and Delorme, A. (2019) Psychophysical Effects in Double-slit Interference Patterns: Response to a Critique. Version 4. https://psyarxiv.com/9csgu/

Radin, D., Wahbeh, H., Michel, L., and Delorme, A. (2020) Commentary: False-Positive Effect in the Radin Double-Slit Experiment on Observer Consciousness as Determined with the Advanced MetaExperimental Protocol. Front. Psychol. 11:726. https://www.frontiersin.org/article/10.3389/fpsyg.2020.00726 
Rubin, M. (2017). Do $p$ values lose their meaning in exploratory analyses? It depends how you define the familywise error rate. Rev. Gen. Psychol. 21, 269-275. doi: 10.1037/gpr0000123

Tremblay, N. (2019). Independent Re-analysis of Alleged Mind-matter Interaction in Double-slit Experimental Data. PLOS One 14:e0211511. DOI: 10.1371/journal.pone.0211511

Tukey, J. W. (1991) The Philosophy of Multiple Comparisons. Statistical Science 6, 100-16.

Von Stillfried, N. and Walleczek, J. (2019) Sham-experiments Reveal a Statistical Error and the Need for Confirmatory Research in the Radin Double-Slit experiment. https://osf.io/nyq5x/

Wagenmakers, E.-J., Wetzels, R., Borsboom, D., and van der Maas, H. L. J. (2011) Why Psychologists Must Change the Way They Analyze Their Data: The Case of Psi: Comment on Bem (2011). J. Pers. Soc. Psychol. 100, $426-432$.

Walleczek, J. and von Stillfried, N. (2019) False-Positive Effect in the Radin Double-Slit Experiment on Observer Consciousness as Determined with the Advanced Meta-Experimental Protocol. Front. Psychol. 10:1891. https://doi.org/10.3389/fpsyg.2019.01891

Walleczek, J. and von Stillfried, N. (2020) Response: Commentary: False-Positive Effect in the Radin Double-Slit Experiment on Observer Consciousness as Determined with the Advanced MetaExperimental Protocol. Front. Psychol. 11:596125. https://doi.org/10.3389/fpsyg.2020.596125 